\title{
Experimental investigation of two-dimensional delamination in GFRP laminates
}

\author{
Aida Cameselle-Molares, Anastasios P. Vassilopoulos, Thomas Keller ${ }^{*}$ \\ Composite Construction Laboratory (CCLab), École Polytechnique Fédérale de Lausanne (EPFL), \\ Station 16, Bâtiment BP, CH-1015 Lausanne, Switzerland
}

\begin{abstract}
The 2D delamination behavior of composite laminates under quasi-static out-of-plane opening loading has been experimentally investigated. A circular embedded pre-crack was introduced in the center and at the midplane of the laminates. To investigate the effect of the fiber architecture on the fracture behavior, six different types of fabrics were selected. Increasing load-displacement curves were obtained due to the increasing crack front length during propagation. During the loading process, stiffening and softening mechanisms were activated. The stretching of the laminates, constituted the main stiffening mechanism that appeared and increased as the plate opened. Once the crack started growing, a corresponding softening due to crack propagation occurred together with a secondary stiffening mechanism, fiber bridging. These stiffness-related mechanisms were reflected in the compliance and clearly differentiate 2D- from 1D-delamination.
\end{abstract}

Keywords: 2D delamination; laminates; embedded pre-crack; crack area.

\section{Introduction}

Composite materials such as GFRPs (glass fiber-reinforced polymers) are commonly used for high performance load-bearing structural elements because of their high stiffness- and strength-to-weight ratios and great resistance to environmental impact (e.g. corrosion) [1]. The successful use of FRP for primary structural parts depends on its integrity and reliability. Delamination in laminated composites, which is likely to occur under compression, out-of-plane or shear loads, is one of the most common and critical types of damage. The relatively weak interlaminar strengths of laminates makes this failure mode particularly important for the integrity of composite structures. Due to the significant reduction in the load-bearing capacity of the structure caused by delamination, many experimental, analytical and numerical investigations have been carried out to characterize the fracture behavior of composite materials [1-6]. Research efforts have focused on beam-like specimens where the crack propagates with an approximately constant width of the crack front only in the longitudinal direction, i.e. one dimensional (1D) crack propagation occurs. However, delamination/debonding damage in real

\footnotetext{
${ }^{*}$ Corresponding author. Tel: +41216933226; email: thomas.keller@epfl.ch
} 
structures is not restricted to one direction but spans all around its contour. Few experimental or numerical investigations emulating the two-dimensional propagation of fully embedded pre-cracks in composite laminates or at the bonding interface between two adherends have been carried out.

2D delamination under shear modes was investigated in [7] for carbon/epoxy laminates under threepoint bending. An elliptical pre-crack was placed at the center and at the midplane of the laminate between two unidirectional $\left(0^{\circ}\right)$ plies. The fracture toughness along the crack front and the failure load were calculated analytically while only the latter was measured. Crack migration along the transverse direction was reported, causing an uneven propagation of the crack.

When damaged FRP sandwich structures are subjected to compressive loads, they tend to exhibit wrinkling of the thin laminates surrounding the damage, leading to the delamination under opening and shear modes $[8,9]$. Most of the work developed in this wrinkling-driven fracture framework is also restricted, as for standardized fracture procedures, to one-directional damage propagation $[9,10]$.

Several works have investigated the two-dimensional buckling and delamination in composite laminates under in-plane pure compression [11-15]. An analytical model able to predict critical values of applied strain preceding the two-dimensional crack propagation due to buckling is presented in [11]. Experimental and numerical analyses of CFRP (carbon fiber-reinforced polymer) laminates under pure compressive load with a circular eccentric delamination were used to evaluate the accuracy of the model. Intra-ply cracking occurred along the directions perpendicular to the fibers, as in [7], and only initiation values could be evaluated in this study. The value of the total critical strain energy released rate (SERR) used in the model was obtained from an energy-based damage propagation criterion. The critical SERR values of pure fracture modes used in this criterion were obtained from standard fracture specimens. Further analytical, numerical and experimental investigations can be found in [12-15]. However, the fracture mechanics parameters used in combination with the analytical and numerical formulations developed for 2D scenarios were again derived from 1D standard experiments.

A numerical study of the two-dimensional fracture behavior of flat and curved honeycomb sandwich panels containing a circular disbond at one of the face sheet/core interfaces is conducted in $[16,17]$. Two loading conditions were considered: in-plane loads and ground-air-ground pressurization. The SERR was evaluated along the disbond using the virtual crack closure technique (VCCT). Standard 1D fracture mechanics specimens were also used to determine the critical SERR of the 2D laminate.

Experimental work to determine the Mode I critical energy release rate $\left(G_{I c}\right)$ under external out-of-plane opening loading at the interface of two circular plexiglas (PMMA) plates bonded together with epoxy was presented in [18]. A circular pre-crack was inserted at the center of one interface and a tensile load was applied to propagate the pre-crack. An expression to calculate $G_{I c}$ for this configuration was developed. The flexural rigidity and deflection formulations at the center of isotropic plates were used to obtain the expression for the compliance. Assuming that the perimeter of the circular crack area (i.e. 
$b=2 \pi a, a$ being the radius of the crack) is equivalent to the width of the crack in a standard double cantilever beam (DCB), a closed expression for $G_{I c}$ independent of the dimension of the crack was obtained. The described assumption might be refutable for two reasons: first in the general expression for $G_{I c}$, the compliance is derived with respect to the crack area, and therefore the chosen value of $b$ multiplied by the radius $a$ should give the value of the cracked area, a condition not fulfilled in this case; second, in this type of experiment, the length of the crack front increases as the crack propagates, and it is therefore a variable and cannot be treated as a constant for the derivation of the compliance. To the authors' knowledge, no other experimental investigation of two-dimensional delamination under an external out-of-plane tensile load has been reported.

In this paper, the main objective was the experimental investigation of the two-dimensional (2D) delamination behavior in GFRP/epoxy plates and to identify potential differences in the behavior compared to 1D delamination. For that purpose, a novel design and experimental set-up suitable for the investigation of the 2D delamination behavior of laminates with internal circular disbonds, subjected to opening loads, similar to the loads applied in a Mode I DCB specimen, was developed. Tensile experiments were performed to progressively open the crack. The load, introduced via an in-house developed loading system, was measured throughout the experiments as well as the crack propagation in the different directions, which was recorded digitally and visually. Based on the experimental results, the evolution of the crack front and consequently the fracture behavior could be analyzed. To understand the different phenomena observed, an FE simulation up to crack initiation was conducted for one of the plates.

\section{Experimental investigation}

\subsection{Material description}

Six different types of glass fiber reinforcements were used to fabricate the laminates: four types of woven fabrics with different proportions of reinforcement in the warp/weft directions (50/50 (W50.50) of 390-g/ $\mathrm{m}^{2}$ weight provided by Swiss-Composite, Switzerland, and 60/40 (W60.40), 70/30 (W70.30), and 80/20 (W80.20) of 500, 510 and 400-g/ $\mathrm{m}^{2}$ weight respectively, supplied by Tissa Glasweberei AG, Switzerland); a long continuous filament mat (CFM) with a weight of $600-\mathrm{g} / \mathrm{m}^{2}$ and no binder or stitching, provided by Owens Corning (United States); and a multidirectional (quadriaxial $0 / \pm 45^{\circ} / 90^{\circ}$ ) sewed (not stitched) fabric (MD) of $800-\mathrm{g} / \mathrm{m}^{2}$ weight supplied by Swiss-Composite (Switzerland). Photos of the different reinforcements are presented in Fig. 1. These fiber architectures were selected with two objectives: 1) to obtain different propagation patterns starting from a circular pre-crack, and 2) to propagate the crack at the midplane and thus avoiding crack migration phenomena (i.e. preventing the crack from jumping between layers) which would complicate the understanding of the results.

The selected matrix was an epoxy resin (Sika Biresin CR83) intended for infusion techniques and supplied by Global Tool Trading AG, Switzerland. The combination of this resin with the selected 
reinforcements resulted in highly translucent laminates for the case of CFM and medium translucent laminates for the remainder, this being an important requirement for this investigation where the crack propagates inside the plate and visual access to the crack front is essential. The properties of the glass fibers and resin are reported in Table 1 (manufacturer data [19-22]). All the reinforcements consisted of E-glass, except for the CFM which was made of E-CR (corrosion resistant) glass.

\subsection{Specimen description and elastic properties}

The experimental program was conducted on twelve GFRP plates, two for each type of reinforcement. The pairs of plates were labeled accordingly (i.e. W50.50.1/2, W60.40.1/2, W70.30.1/2, W80.20.1/2, CFM.1/2 and MD.1/2) and their layup and geometrical description are presented in Table 2.

The elastic properties of the laminates were estimated based on the fiber volume fraction, calculated as $f=w /\left(\rho_{f} d\right)$, where $w$ is the weight of the reinforcement, $\rho_{f}$ the density of the glass fibers and $d$ the thickness of the plate. For the woven (W) laminates, the in-plane E-moduli were calculated using Eq. (1) $[23,24]$ :

$E_{1,2}=m_{1,2}\left(E_{r}+\left(E_{f}-E_{r}\right) f+E_{90}\right.$

where $m_{1,2}$ are parameters indicating the proportion of reinforcement for each direction (i.e. 0.5 for both directions in W50.50); $E_{r}$ and $E_{f}$ are the E-moduli of the resin and fiber respectively; $f$ is the fiber volume fraction and $E_{90}$ is the E-modulus in the $90^{\circ}$ direction of a unidirectional layer with the same fiber volume fraction as the woven plate calculated by the Halpin-Tsai formulation [24]. The out-ofplane E-modulus $E_{3}$ equals $E_{90}$. The in-plane E-modulus for the continuous filament mat laminates (CFM) was calculated according to Eq. (2) [25]:

$E_{1}=E_{2}=\frac{3}{8} E_{0}+\frac{5}{8} E_{90}$

where $E_{0}$ and $E_{90}$ are the E-moduli in the $0^{\circ}$ and $90^{\circ}$ directions of a unidirectional layer with the same volume fraction as the CFM plate. The out-of-plane E-modulus was assumed to be the same as for the woven laminates. The Poisson's ratios for the CFM laminates were extracted from the database in [23] and for the woven laminates Eq. (3), Eq. (4) and Eq. (5) were used [23]:

$v_{12}=\left(v_{r}-\left(v_{r}-v_{f}\right) f\right) m_{1}\left[1+\frac{E_{90}}{E_{r}+\left(E_{f}-E_{r}\right) f}\right]$

$v_{13}=\left(v_{r}-\left(v_{r}-v_{f}\right) f\right) m_{1} f$

$v_{23}=\left(v_{r}-\left(v_{r}-v_{f}\right) f\right) m_{2} f$

where $v_{r}$ and $v_{f}$ are the Poisson ratios of the resin and fiber. Finally, the shear modulus of the woven laminates was calculated using Eq. (6), Eq. (7) and Eq. (8) [23, 24]:

$G_{12}=G_{r} \frac{1+\zeta \mu f}{1-\mu f}$ 
$G_{13}=G_{r} \frac{1+\zeta \mu f m_{1}}{1-\mu f m_{1}}$

$G_{23}=G_{r} \frac{1+\xi \mu f m_{2}}{1-\mu f m_{2}}$

where $G_{r}$ is the shear modulus of the resin, $\zeta=2$ for fibers of circular cross-section and $\mu$ is a parameter calculated with Eq. (9):

$\mu=\left(\frac{E_{f}}{E_{r}}-1\right) /\left(\frac{E_{f}}{E_{r}}+\zeta\right)$

The shear modulus $G_{12}$ of the CFM laminates was calculated with the general isotropic material formulation and $G_{13}$ and $G_{23}$ were calculated using the same formulation as that indicated for W50.50 laminates. There is no empirical formulation available to determine the elastic properties of MD specimens. Therefore, Composite Laminate Theory (CLT) was used to approximate $E_{1}, E_{2}, G_{12}, v_{12}$ values and the same procedure as for the woven laminates was followed to calculate the out-of-plane properties. The obtained values of one plate of each type are presented in Table 3.

All the GFRP laminates were symmetric with respect to both the midplane and each of the halves separately. Likewise, they were all fabricated by a vacuum infusion process. The plate configuration is shown in Fig. 2 and the fabrication procedure is described in the following. First, three layers of release agent were applied over the polypropylene substrate to facilitate the demolding of the plates. Then half of the reinforcement layers (i.e. up to the midplane) were placed and lightly fixed with tape to the substrate. To introduce the load in a distributed manner, two stainless steel inserts of 1.2-mm thickness and 100-mm diameter were placed at the center of the reinforcements. Between them, a Teflon film of $13-\mu \mathrm{m}$ thickness and 180-mm diameter was placed to introduce the pre-crack (see Fig. 2). To ensure that both steel inserts and the Teflon remained vertically aligned during the infusion process, preventing them from sliding, a very small pin was welded at the center of one of the steel plates and a small hole was drilled into the other so that a local shear bearing connection was created and no relative movements between them occur during the infusion process. Finally the remaining layers were placed and fixed and the whole system was infused. The thicknesses of the plates around the inserts were slightly greater than for the rest of the laminate. Their sufficient distance from the crack front is enough to make this difference unnoticeable with regard to the fracture behavior.

The infusion was performed from side to side of the specimens. To obtain a good impregnation of the entire laminate, especially around the steel insert where the flow is particularly impeded, the infusion speed was carefully regulated. Likewise, for the multiaxial and woven reinforcements whose fiber architecture makes them harder to impregnate, flow meshes were placed above and below the plates and were connected to their own resin inlet. The plates with continuous filament mat reinforcement only required the upper flow mesh with the corresponding resin inlet. To eliminate the air inside the resin before the infusion, a degassing process was carried out. The laminates were cured under vacuum for at 
least 16 hours at room temperature and subsequently, once demolded, they were cured at $70^{\circ} \mathrm{C}$ for eight hours.

The procedure developed for the introduction of the out-of-plane load into the infused plates is detailed in Fig. 3, with the different steps of the procedure indicated with the numbers in red color. First, six holes of $\varnothing 10 \mathrm{~mm}$ were drilled starting from the bottom side of the plate until the midplane, where the diameter was changed to $\varnothing 6 \mathrm{~mm}$ to complete the hole from the midplane to the other side of the plate (Fig. 3 (c), Steps 1 and 2). The same procedure was followed from the top side but with an alternated location of the holes (i.e. again six holes but rotated by $30^{\circ}$ with respect to the center). All the holes were drilled along a radial length of $35 \mathrm{~mm}$ from the center of the plate, delimiting thus the loading line (see Fig. 2). Subtracting the radial length of the loading line from that of the Teflon (i.e. $90 \mathrm{~mm}$ ) resulted in a pre-crack of $55 \mathrm{~mm}$ radial length. Six downward facing screws were then placed followed by six upward facing ones (Fig. 3 (c), Step 3). The latter were immediately tightened to an 8-mm thickness and 100-mm diameter upper steel plate (Fig. 3(c), Steps 5 and 6), which was pierced in advance with six holes of $\varnothing 6$ and an additional centered hole for a screw of $\varnothing 8 \mathrm{~mm}$. This central screw was also inserted in advance (Step 4) and intended for the assembling of a piano hinge (see Fig. 3 (a)) that connected the specimen to the grips of the machine. Finally the downward facing screws were also tightened to their corresponding lower steel plate and the piano hinges were assembled. The piano hinges assured that any small specimen rotation would not damage the loading cell of the machine. Consequently, the centrally applied load was distributed by the (sufficiently stiff) upper and lower external steel plates to the upper and lower groups of six screws, which opened the system via the internal steel plates, as shown in Fig. $3(d)$.

\subsection{Experimental set-up, instrumentation and measurements}

The experiments were performed under displacement-control on a $\mathrm{W}+\mathrm{B}$ electromechanic machine of $50-\mathrm{kN}$ capacity at a rate of $1.5 \mathrm{~mm} / \mathrm{min}$, a temperature of $24 \pm 2^{\circ} \mathrm{C}$ and relative humidity of $38 \pm 5 \%$. The experimental set-up and instrumentation layout are shown in Fig. 4. Once the load-introduction system was assembled, the plate was placed and fixed within the grips of the machine, by adjusting first the bottom hinge and then, with the help of a level so that the plate is completely horizontal, the upper hinge.

Due to the nature of the experiment and difficulty of measuring the entire contour of the crack, three different measuring systems were employed: the 3D Digital Image Correlation System (DIC), a digital camera and visual measurements. Eight rulers starting from the end of the pre-crack were drawn on the plates corresponding to eight directions of propagation, named after the cardinal directions (see Fig. 5). The propagation along the west ("E") direction was measured by the DIC system (accuracy of \pm 0.005 $\mathrm{mm}$ ). Due to the set-up requirements, the two DIC cameras were placed below the plate (Fig. 4 (b)). A random $\leq 0.1 \mathrm{~mm}$ speckle pattern using black spray paint was applied over the surface (see Fig. 4). The 
ruler corresponding to this direction, drawn on the bottom surface, was slightly different from the others as small black points $2.5 \mathrm{~mm}$ apart were used instead of continuous lines so that the general DIC pattern was not disturbed (Fig. 5). The calibration area around this dotted line was approximately $50 \mathrm{~mm}$ wide and $100 \mathrm{~mm}$ long. In order to obtain clear, good quality images, a source of white light was employed with a semi-opaque diffusor. The set-up was the same for all the plates except for the CFM laminates, where a layer of white paint was additionally applied before the black speckle pattern due to the high transparency of these specimens causing reflections and affecting the quality of the measurements during the experiment. The acquisition frequency of the images was set to $0.2 \mathrm{~Hz}$. The processing of the results was carried out using Vic-3D software from Correlated Solutions Inc. [26]. In Fig. 6 (a) an example of the measuring area and the selected path of interest are presented. The path is superposed on the dotted ruler and therefore starts at the end of the pre-crack in that direction. The values of the outof-plane displacements were extracted along the path and the crack tip was defined by means of a numerical script as the point where the deformed profile of the path changes from straight to curved. An example of the profiles obtained for the plate W50.50.1 is presented in Fig. 6 (b) and the corresponding load and displacement values for the indicated crack tips can be found in Fig. 7 (a). Positive values correspond to deformation towards the location of the DIC cameras and negative values to the opposite direction. During deformation, two contributions to the out-of-plane displacements were measured by the DIC system: the solid rigid movement of the whole plate in the negative direction (i.e. upwards together with the loading frame of the machine) and the out-of-plane deformation due to the opening of the crack in the positive direction. Therefore, as a result of the positive contribution of the opening of the crack and as can be observed in Fig. 6 (b), the out-of-plane displacement values in the cracked region (i.e. curved part of the profiles) are smaller in absolute terms than in the non-cracked region. The obtained results were cross-checked with the corresponding values observed in the DIC pictures by means of the drawn dotted ruler and a very good agreement was found.

The direction of propagation monitored with the digital camera was either north ("N") or northwest ("NW") depending on the type of reinforcement and the illumination requirements. The diagonal "NW" direction was selected for W50.50 plates providing that the crack was expected to propagate along the orthogonal directions equally and one of them, the "E" direction, was already recorded by the DIC. For the rest of the woven plates, where the crack propagated differently along the two orthogonal directions, the "N" direction was monitored. The illumination affected the measurements of the CFM plates whose transparency made it hard to measure the " $\mathrm{N}$ " direction with the digital camera due to the high intensity of the reflections in the pictures. Therefore, the "NW" direction was selected. The images were taken from above the plate (see Fig. 4 (b)) and consequently the rulers were drawn on the upper surface of the plates. The acquisition frequency was set as for the DIC to $0.2 \mathrm{~Hz}$, and each picture was registered with the corresponding values of the load and displacement. Post-processing visual work based on the images 
was performed. The type of image obtained by the camera during the experiment can be observed in Fig. 4 (b, "Digital Camera Sample Image").

Along the remaining directions, where the rulers were also drawn on the upper surface, the crack front was recorded visually. Every time the crack propagated $2.5 \mathrm{~mm}$, measured over the rulers, the values of the crack front along all these directions were registered together with the load and the displacement.

To record the boundary movements, four Linear Variable Differential Transducers (LVDTs, accuracy of $\pm 0.02 \mathrm{~mm}$ ) were installed (Fig. 4 (b)) to measure the displacement of the four corners. The load and the displacement at the center of the plates (out-of-plane) were obtained from the machine (accuracy of $\pm 0.11 \%)$. The whole experiment was likewise recorded with a video camera.

\section{Experimental results}

\subsection{Load-displacement responses and crack propagation measurements}

Continuously increasing load-opening displacement curves were obtained for all the experiments, also after crack initiation (Fig. 7 to Fig. 11). For plates W50.50, W60.40 and MD, the experiments were stopped after the load started to decrease. For plates W70.30, CFM and W80.20, the crack propagation was automatically interrupted by the matrix failure and therefore a sharp decrease in the loads can be observed in Fig. 9, Fig. 10 and Fig. 11 (b), respectively. The values of the maximum load and displacement achieved for each specimen are presented in Table 4.

As mentioned in the previous section, the vertical displacements on the four corners were recorded by the LVDTs. Different values were recorded depending on the corner for all the plates except for the CFM pair, where the four corners presented approximately the same displacement values. One of the reasons for these differences in the vertical displacements of the boundaries of the plates was the variation in stiffness depending on the direction of propagation. As a result, the crack tip along the stiffest directions (e.g. the orthogonal directions for W50.50) exhibited the greatest opening and therefore a greater propagation and vertical movement of the un-cracked region in that direction. Also, depending on the propagation direction, distances from the crack tip to the free end were different (longer in the diagonal and shorter in the orthogonal directions), also affecting the magnitude of these vertical movements: the further the free end from the crack tip, the greater the vertical displacement. The combination of both effects led to the recorded boundary behavior, unique for each type of reinforcement. Initially, the crack propagations were not affected by these boundary displacements and a symmetric and stable propagation could be observed. When the boundary displacements became higher, they started to influence the crack front and the symmetry in the growth was lost (see Fig. 12). The limits of the symmetric behavior were obtained based on the data registered during the experiment (i.e. during the experiments, load, displacement and crack length measurements along all the directions were noted when the loss in symmetry was noticed). A subsequent revision of all the digitally recorded data was performed to corroborate the in-situ registered data. As an example, the obtained curve of 
corner and opening displacements vs the load for specimen W50.50.1 is shown in Fig. 13. It can be seen that for this type of plate the corners exhibited a similar behavior in a two-by-two trend, with the displacement of the "NW"/"SE" pair being higher than for the "NE"/"SW" pair. The opening displacement at the limit was $30 \mathrm{~mm}$ and is indicated with a red dashed line. In this case, from the limit onwards, the displacements of the corners became unstable, and the measurements for the "NW"/"SE" pair were lost.

The advance of the crack front was measured up to the end of the experiments for all the plates. However, only the results concerning plates W50.50, W60.40, W70.30 and CFM are described in the following since the results obtained for the W80.20 plates (Fig. 11 (b)) were very similar to those for W70.30 and the output obtained from the MD plates (Fig. 11 (a)) was not conclusive due to the crack jumping and the consequent asymmetry in the propagation. A further description of the crack propagation patterns will be given in Section 3.2.

The crack lengths vs opening displacement responses of the W50.50 pair are shown in Fig. 7. Crack length values in the "NE" direction for W50.50.1 are missing due to technical problems with the digital camera during the experiment and for W50.50.2, which was the first specimen of woven reinforcement investigated, only the "E", "NW" and "S" directions were measured, the first two by digital means (DIC and digital camera respectively) and the third visually. For the remainder of the plates the full set of measurements was obtained. The blue lines represent the orthogonal directions (i.e. $0 / 90^{\circ}$, both equally reinforced in this case) and the magenta lines represent the diagonal directions (i.e. $\pm 45^{\circ}$ ). It can be observed that the measured lengths along the diagonal directions remained fairly consistent with each other as well as for the orthogonal directions, indicating a practically symmetric growth of the crack front. The limit of the symmetric behavior corresponded, as previously indicated, to a 30-mm opening displacement and is marked with a dashed vertical red line. It is evident in Fig. 7 (a) how the values between the diagonal crack lengths and also between the orthogonal crack lengths began to diverge from this point onwards. Additionally, in order to obtain a continuous evaluation of the crack front, average curves for the orthogonal and diagonal directions were derived by means of parabolic expressions, as shown in Fig. 7.

The same representation is shown in Fig. 8 for the W60.40 plates. Here, the blue lines represent the orthogonal directions with $60 \%$ reinforcement, the green lines the orthogonal directions with $40 \%$ of reinforcement and the magenta lines the diagonal directions. Some sudden crack propagations occurred in the case of the W60.40.2 specimen in the $60 \%$ directions as can be observed in both the load and crack length curves (sharp variations) showing a subsequent very rapid recovery of the load. The corresponding average crack length curves were also plotted for the three groups of directions. The limit of symmetric propagation in this case corresponded to a displacement of $26.2 \mathrm{~mm}$ also indicated with a red vertical dotted line. It can be observed that, as expected, it was along the most reinforced directions 
(blue lines) that the crack front propagated the furthest. Initially, the propagation along the less reinforced directions was slightly greater than along the diagonals. Once the crack fronts advanced sufficiently further, the behavior was inversed and the propagation along the $40 \%$ reinforced directions became smaller (see Fig. 8).

The results obtained for plates type W70.30 are shown in Fig. 9. As for the W60.40, the three main directions are represented in blue (orthogonal of 70\%), green (orthogonal of 30\%) and magenta (diagonals). The average curves are also added for each direction. This type of laminate exhibited a less smooth crack propagation than the previous plates, with two sudden propagations in the most reinforced directions for W70.30.1 (Fig. 9 (a)) and three for W70.30.2 (Fig. 9 (b)), also showing a subsequent rapid recovery of the load. It can be observed how the crack barely propagated along the least reinforced direction. In this case, the crack did not propagate far enough to lose the symmetrical crack shape.

The load and crack lengths vs the displacement curves for CFM plates are presented in Fig. 10. Due to the concentric growth of the crack up to the failure of the matrix near the holes, the values of the crack lengths showed the same trend. The CFM.2 plate exhibited two small sudden crack propagations reflected in both the load and crack lengths (Fig. 10 (b)) followed by a rapid recovery.

The initiation points are indicated for all the plates in Fig. 7 to Fig. 10 and the corresponding load and displacement values are given in Table 4. As can be observed in the figures, no specific change in the load could be noticed for most of the plates except for the W60.40 pair and CFM.1 where a small load fluctuation was registered. The values were obtained in situ during the experiments (i.e. when the crack propagation was initiated, the load and displacement values were noted) and a subsequent revision of all the digitally recorded data (pictures from digital camera and DIC) was performed to corroborate them.

\subsection{Crack propagation patterns}

The different crack propagation patterns obtained are shown in Fig 14-19. All these pictures were taken with a black background in order to improve the contrast and better identify the propagated crack area except for the CFM plates where, due to their high translucency, a white background was selected.

Crack propagation in the W50.50 plates advanced symmetrically to the orthogonal axes up to around 30 $\mathrm{mm}$ of propagation in the diagonal directions and $50 \mathrm{~mm}$ of propagation in the orthogonal directions in both plates. The shape of the crack front for the last symmetric contour for W50.50.1 is drawn in blue in Fig. 14. From this point onwards, the boundary displacements started to affect the specimens' propagation behavior, impeding the growth of the crack in some directions (e.g. "NE") and favoring others (e.g. "N"). A similar performance was found for the W60.40 laminates where the crack grew symmetrically to a propagation length of around $45 \mathrm{~mm}$ in the orthogonal directions of $60 \%$ reinforcement, $15 \mathrm{~mm}$ in the orthogonal directions of $40 \%$ reinforcement and $20 \mathrm{~mm}$ in the diagonal 
directions. In Fig. 15 the shape of the last symmetric crack front for the plate W60.40.1 is also indicated in blue. Plates W70.30 and W80.20 only propagated $2 \mathrm{~mm}$ in the less reinforced directions and 20-30 $\mathrm{mm}$ in the more reinforced directions before they failed due to matrix cracking, starting from the holes (see Fig. 16 and Fig. 17). For the CFM laminates a concentric circular crack front propagation was observed as expected for an in-plane isotropic reinforcement. A propagation along the entire contour of $22.5 \mathrm{~mm}$ and $25 \mathrm{~mm}$ for CFM.1 and CFM.2 respectively was achieved before they also failed as a result of matrix failure initiating from the holes (Fig. 18). The crack initiated and propagated in all of these plates between the adjacent plies of the pre-crack (i.e. the midplane).

For the MD plates the propagation was symmetric only at the very beginning of the experiments (2-3 $\mathrm{mm}$ of propagation) for both plates but, as soon as the crack propagated further, crack migration phenomena occurred all around the contour creating a non-symmetric propagation pattern that was not even repeatable from one plate to the other (Fig. 19). As previously mentioned in Section 2.1, in the MD reinforcement, and unlike in the $\mathrm{W}$ (woven) reinforcement, the different directions were sewed together (each direction one layer). Given the results, it is evident that this sewing was not sufficient to join the layers firmly together, i.e. enable them to behave as a single layer and permit the crack to propagate in the plate's midplane. Therefore the crack started to migrate from one layer to another depending on the direction of propagation as might have occurred for a laminate with unidirectional reinforcements stacked in different directions.

Based on the derived average curves of crack-length along the different directions of propagation vs the opening displacement (see Fig. 7 to Fig. 10) a back-calculation of the crack front was performed for each increment of the experiment. The advance of the crack front is represented for three of the plates, W50.50.1 (Fig. 20 (a)), W60.40.1 (Fig. 20 (b)) and CFM.1 (Fig. 20 (c)). The last contour corresponds to the last crack front before the loss of symmetry for the woven plates (also specified as previously indicated with a blue contour in Fig. 14 and Fig. 15). Correspondingly, the last contour of the CFM is the last before the failure of the matrix. It can be observed how the average curves are able to reproduce the symmetry with respect to the orthogonal axis shown in the experiments.

\subsection{Compliance behavior}

As has been described, the experimental values of the crack lengths along the different directions were used to derive the corresponding average curves, providing continuous evaluation of the crack propagation fronts. The area of the crack was calculated for each increment as the area between the obtained crack front and the loading line (as for the standard fracture mechanics experiments), i.e. a 70$\mathrm{mm}$ diameter circle passing through the center of the screws that constitute the loading points (see Fig. 2). The compliance, calculated as $\delta / P, \delta$ being the opening displacement and $P$ the load, plotted against the crack area is shown for W50.50 (Fig. 21), W60.40 (Fig. 22), W70.30 (Fig. 23) and CFM (Fig. 24). The crack initiation points are indicated. Woven laminates W50.50 and W60.40 exhibited comparable 
behavior, i.e. first a descending branch down to a minimum and then an ascending branch corresponding respectively to a stiffening and subsequent softening of the system. Only the first descending part was developed for the W70.30 plates (Fig. 23) where the crack propagation was significantly less than in the other plates. The W80.20 specimens behaved in the same manner. For the CFM plates, a third branch appeared, apart from the descending and ascending parts already described for the previous plates, showing an increase in the compliance during the initial propagation of the crack (Fig. 24).

\section{Discussion}

\subsection{Increasing load behavior}

As described in Section 3.1, a continuously increasing load-opening displacement behavior was monitored from the beginning of all experiments up to the loss of symmetry of propagation or failure of the specimen, depending on the plate. In contrast to the results shown in this paper, in standard fracture mechanics experiments, the load always decreases once the crack starts to propagate. This contradictory behavior can be understood by comparing how the crack grows in both cases. For fracture mechanics experiments, or any beam-like experiment where the crack width is constant and the length of the crack front also remains practically constant, the crack area grows proportionally throughout the entire experiment. For the two-dimensional experiments presented here, the crack was embedded inside the laminate and consequently, since the crack area started to grow symmetrically with respect to the orthogonal axes, the length of the crack front increased accordingly. As a result, a disproportionate growth of the crack area occurred, whose increments were higher as the crack advanced, forcing the load to increase in order to continue the propagation of the crack.

When the boundary displacements started to affect the propagation of the crack, the length of the crack fronts ceased to increase symmetrically. Some of the directions of the crack fronts were constrained by the boundary deformation of the plate and some other directions were favored (see "W" in Fig. 15 (W60.40.1)). This caused a reduction of the crack front length and consequently a decrease in the load (Figs. 7, 8 and 11 (a)).

\subsection{Stiffness-related mechanisms}

Three different mechanisms affecting the specimens' stiffness could be distinguished in the experiments: stretching, fiber bridging and crack propagation. When thin beams, plates or shells are subjected to transversal efforts and their boundary conditions are fixed on both sides in such a way that their deformation will cause in-plane tension, the structure will become significantly stiffer with increasing deformation [28]. This phenomenon is called stretching and is normally disregarded for standard fracture mechanics experiments as it barely affects the propagation of the crack. For the specimens investigated here, the boundary conditions caused stretching to become a significant stiffening mechanism. As the crack opened under the out-of-plane tensile load, two curvatures appeared: the radial and the circumferential, see Fig. 12. The frame-like boundary of the plates prevented the plate 
from moving radially (i.e. horizontally), causing the corresponding radial stretching. Likewise, the embedded geometric nature of the crack prevented the plate from moving in the circumferential direction, causing the corresponding circumferential stretching. The plates were therefore subjected to a bi-directional stretching phenomenon. Once the crack started propagating, two additional mechanisms were activated, fiber bridging, as an additional stiffening mechanism, and crack propagation itself as a softening mechanism. The fiber bridging was not measured and due to the nature of the experiment it could not be observed either, but it was expected to exist mainly for the CFM specimens and on a much smaller scale for the rest of the specimens. Typically, for a standard fracture experiment, crack propagation is the dominant mechanism after crack initiation, leading to a continuous upwards behavior of the compliance. On the contrary, for these two-dimensional experiments, the stiffening mechanisms (mainly stretching) played an important role in the performance of the specimens, prevailing over the softening due to crack propagation and thus significantly affecting the results. Since the experimental compliance results did not correspond uniquely to the propagation of the crack as for standard fracture specimens, the fracture resistance cannot be directly derived from the experiments.

Based on the curves presented in Fig. 21, an identification of the stiffening and softening regions in both the compliance vs crack curve (for W50.50.1) and load vs displacement curve (for both W50.50 specimens) was carried out in Fig. 25. Two regions were differentiated on the compliance vs crack area curve. The first region, designated " $A$ ", which spans from crack initiation (called "initiation point") up to a minimum (called "transition point"), represents the interval where the stiffening mechanisms (stretching and fiber bridging) prevail over the softening mechanisms (crack propagation), causing the corresponding decrease in compliance. Only when the softening due to crack propagation becomes the dominant mechanism, prevailing over the others (at the transition point) does the compliance start to grow, then entering the second region, designated "B" (Fig. 25(a)).

The same differentiation procedure in the compliance vs crack curve can be established for the W60.40, W70.30 and CFM plates in Fig. 22, Fig. 23 and Fig. 24 respectively by identifying the minimum values as from which the general trend of the compliance increases. Only a part of region A could be identified in Fig. 23, signifying that for plates W70.30 the crack did not propagate sufficiently to become the dominant mechanism (i.e. the failure of the matrix occurred before the transition point was reached). For the CFM plates (Fig. 24), an initial ascending part of the compliance could be observed, which was attributed to isolated initial slipping at the loading blocks and this part was therefore not considered in the further analysis. All the regions and points of interest were likewise identified in the corresponding load-displacement curves for the W60.40 (Fig. 26) and CFM (Fig. 27) plates.

Based on the compliance vs crack area curves, the thresholds indicating initiation of crack propagation were plotted with dashed lines for each pair of plates and the thresholds representing the transition from a dominant stiffening behavior to a dominant softening behavior were plotted with dotted lines. It is 
evident how the stiffening zones corresponded to a continuous increase of the load vs displacement slope and correspondingly, how the softening zones following the transition points corresponded to a decrease of the slope.

In order to confirm the presence of a stretching mechanism and its contribution to the stiffening of the plates, an FE model using the commercial finite element analysis (FEA) software Abaqus 6.14.1 was developed (Fig. 28). The plate chosen for the model development was the W50.50.1, and a total displacement corresponding to the experimental value of initiation was applied, i.e. the behavior before crack propagation was simulated. Based on the boundary movements, the diagonals of the plate were taken as the two axes of symmetry and only one quarter of the model was built. The steel inserts and the external loading blocks were not modeled. Instead, the nodes of the inner faces of the loading areas were tied together with a rigid body condition to reference nodes where the boundary conditions were applied. The built-in continuum shell elements of eight nodes and reduced integration (CS8R) from Abaqus/Standard [27] were used. The mesh comprises 75146 nodes and 56664 elements, with two elements through the thickness of each of the halves above and below the midplane half plate. The size of the mesh varied from $0.6 \mathrm{~mm}$ close to the crack to $4 \mathrm{~mm}$ far from the crack. A static standard calculation taking large displacements into account was carried out. The engineering constants employed to define the material are presented in Table 3. The stress results are plotted in a cylindrical coordinate system and the values in the radial and circumferential directions are shown in Fig. 28 (a) and Fig. 28 (b) respectively. The values at a specific through-thickness section (indicated as "extraction section" in Fig. 28), located at $\rho=68 \mathrm{~mm}$ and $\varphi=\pi / 4$, were extracted and the stretching stresses were calculated in both directions by subtracting the bending stresses from the total stresses obtained. The result of the stretching stresses vs the load is presented in Fig. 29. In can be observed that the stretching in the circumferential direction grows slightly faster than in the radial direction, reaching a proportion of $55 \% / 45 \%$ (circumferential/radial) at the end of the simulation. Consequently, as the plates deformed under the out-of-plane loading, they were subjected to bending and stretching stresses, the latter causing a considerable double stiffening effect capable of retarding the softening of the system when the crack started to propagate.

\section{Conclusions}

The 2D delamination behavior of composite laminates with a circular embedded pre-crack under quasistatic out-of-plane loading has been experimentally investigated. Different fiber architectures were selected to obtain different propagation patterns starting from a circular pre-crack. Two plates of each type were investigated and the crack propagation modes and load-bearing and compliance behavior were analyzed. Furthermore, an FE model corresponding to one of the plates was developed in order to study the stretching effects and their contribution to the stiffening of the system. The following conclusions were drawn: 
1. An experimental design suitable for investigating the $2 \mathrm{D}$ propagation of an embedded pre-crack under out-of-plane opening loading was successfully developed. The different methods employed for the measurement of crack propagation were consistent and reliable.

2. Increasing loads, also after crack initiation, were obtained as a result of a continuously increasing crack front length and a consequently disproportionate increase in the propagation area. This is intrinsic to an embedded crack that grows all around its contour.

3. As the plates started to deform under the out-of-plane loading, stretching stresses appeared in both the radial and circumferential directions as a result of the geometrical constraints. Consequently, the plates were subjected to a dual stiffening effect.

4. Stretching of the specimens and also fiber bridging mainly for the CFM specimens (both stiffening mechanisms) were capable of delaying the general softening of the system that typically occurs once the crack started to propagate. The threshold indicating the point where softening due to crack propagation became the dominant mechanism could be clearly identified as the minimum in the compliance vs crack area curves.

5. Regarding the type of reinforcement, different propagation modes and patterns were obtained, the most reinforced direction being the one experiencing the greatest crack propagation. In the woven and continuous filament mat reinforcements, the crack remained in the mid-plane and propagated symmetrically with respect to the orthogonal axes, whereas for the multiaxial reinforcement (nonwoven) crack migration effects occurred and non-symmetrical propagation was observed.

6. Out-of-plane displacements of the boundaries of the plates were measured for all specimens as a result of the different rates of crack propagation and distances to the free end along the crack front. The propagation of the crack was unaffected by these boundary effects up to a recognizable limit. From this limit onwards, the results were not considered.

7. The FE model developed for one of the woven plates simulating the experimental behavior up to crack initiation showed that the stretching stresses in the circumferential direction increased slightly faster than in the radial direction. Significant values were reached in both directions, thus confirming the experimental results, i.e. the activation of this mechanism.

In this paper, experimental results have been presented and discussed and an FE model has been introduced to investigate the stretching mechanisms. Further numerical analysis is being conducted to fully characterize the fracture behavior of the plates beyond crack initiation.

\section{Acknowledgments}

The authors wish to acknowledge the support and funding of this research by the Swiss National Science Foundation (Grant No. 200021_156647/1). 


\section{References}

1. Bakis, C., et al., Fiber-reinforced polymer composites for construction-state-of-the-art review. Journal of Composites for Construction, 2002; 6(2): 73-87.

2. Benzeggagh, M.L., Kenane, M. Measurement of mixed-mode delamination fracture toughness of unidirectional glass/epoxy composites with mixed-mode bending apparatus. Compos. Sci. Technol., 1996; 56: 439-449.

3. ASTM D5528-13: Standard test method for mode I interlaminar fracture toughness for unidirectional fiber-reinforced polymer matrix composites, in Annual book of ATM standards: adhesive section 15.03.

4. ASTM D6671/D6671M - 13e1: Standard test method for mixed mode I-mode II interlaminar fracture toughness for unidirectional fiber-reinforced polymer matrix composites, in Annual book of ATM standards: adhesive section 15.03.

5. Turon, A., Dávila, C.G., Camanho, P.P., Costa, J. An engineering solution for mesh size effects in the simulation of delamination using cohesive zone models. Eng. Fract. Mech., 2007; 74: 16651682.

6. Shahverdi M, Vassilopoulos AP, Keller T. Modeling effects of asymmetry and fiber bridging on Mode I fracture behavior of bonded pultruded composite joints. Eng Fract Mech, 2013; 99: 335348.

7. Chatterjee, S.N., Dick, W.A., Byron Pipes, R. Mixed-mode delamination fracture in laminated composites. Compos. Sci. Technol., 1986; 25: 49-67.

8. Carlsson, L.A., Kardomateas, G.A. Structural and Failure Mechanics of Sandwich Composites, 2011; Solid Mechanics and its Applications vol. 121; Springer.

9. Rasmus, C., Østergaard, C. Buckling driven debonding in sandwich columns. Int. J. Solids Struct., 2008; 45: 1264-1282.

10. Kinawy M., Butler, R., Hunt, G.W. Bending strength of delaminated aerospace composites. Phil. Trans. R. Soc. A, 2012; 370: 1780-1979.

11. Butler, R., Rhead, A.T, Liu, W., Kontis, N. Compressive strength of delaminated aerospace composites. Phil. Trans. R. Soc. A, 2012; 370:1759-1779.

12. Nilsson K.F., Thesken J.C., Sindelar P, Giannakopoulos A.E., Storakers B. A theoretical and experimental investigation of buckling induced delamination growth. J. Mech. Phys. Solids, 1993; 41(4): 749-782.

13. Riccio A., Raimondo A., Di Caprio F., Scaramuzzino F. Delaminations buckling and growth phenomena in stiffened composite panel under compression. Part II: a numerical study. Journal of Composite Materials, 2014; 48(3); 2857-2870.

14. Rhead A.T., Butler R., Hunt G.W. Compressive strength of composite laminates with delamination induced interaction of panel and sublaminate bucking modes. Composite Structures, 2017; 171; 326-334. 
15. Nilsson K.F, Asp L.E., Alpman J.E., Nystedt L. Delamination bucking and growth for delaminations at different depth in a slender composite panel. Int. J. Solid Struct., 2007; 38; 30393071.

16. Chen, Z.M., Krueger, R., Rinker, M. Facesheet/Core Disbond Growth in Honeycomb Sandwich Panels Subjected to Ground-Air-Ground Pressurization and In-Plane Loading. In: 11th International Conference on Sandwich Structures ICSS-11, Ft. Lauderdale, USA, March, 2016.

17. Rinker, M., Krueger, R., Ratcliffe, J. Analysis of an Aircraft Honeycomb Sandwich Panel with Circular Face Sheet/Core Disbond Subjected to Ground-Air Pressurization. NASA/CR-2013217974, 2013.

18. Kumar, P., Reddy, S.R. Experimental determination of interlaminar GIc using a fully embedded centre-cracked specimen. Eng. Fract. Mech., 1998; 59(2): 183-189.

19. Owen's Corning Reinforcements Composite Solutions Guide.

$<$ http://www.ocvreinforcements.com/pdf/library/Composite_Solutions_Guide_100360_E_finalpri ntable.pdf $>$ (Accessed 26 March 2018).

20. Swiss Composite Product Catalogue. $<$ https://www.swisscomposite.ch/pdf/Produkteuebersicht.pdf > (Accessed 16 October 2017).

21. Tissa Glasweber AG website. $<$ http://www.tissa.ch/44Def.aspx?tabindex $=0 \&$ tabid=500\&lang=en $>$ (Accessed 16 October 2017).

22. Sika datasheet product. <https://deu.sika.com/dms/getdocument.get/dd4b783e-bfa5-32e3-a705f9f548f6b326/Biresin_CR83_eng.pdf $>$ (Accessed 19 October 2017).

23. Ascione L, Caron J-F, Godonou P, Van IJselmuijden K, Knippers J, Mottram T, et al. Prospect for new guidance in the design of FRP: Support to the implementation, harmonization and further development of the Eurocodes. Publications Office of the European Union; 2016. Report No. EUR 27666 EN. Doi: http://dx.doi.org/10.2788/22306.

24. Jones R. M. Mechanics of Composite Materials, 1999; 2nd Edition: Taylor and Francis.

25. Gay D., Hoa S.V. Composite Materials, Design and Application, 2007; 2nd Edition: CRC Press, Taylor and Francis.

26. Correlated Solutions Inc. VIC 3D-v7 reference manual. Columbia, USA: Correlated Solutions.

27. Abaqus Inc. Abaqus analysis user's manual, version 6.14-1. 2014. Providence, RI, USA.

28. Howell L.L. Compliant Mechanisms, 2001: John Wiley \& Sons Inc. 
Tables:

Table 1. Material properties

\begin{tabular}{lcccc}
\hline Material & $\begin{array}{c}\mathrm{E} \\
(\mathrm{GPa})\end{array}$ & $\begin{array}{c}\mathrm{G} \\
(\mathrm{GPa})\end{array}$ & $\begin{array}{c}v \\
(-)\end{array}$ & $\begin{array}{c}\rho \\
\left(\mathrm{g} / \mathrm{cm}^{3}\right)\end{array}$ \\
\hline Epoxy resin & 2.96 & 1.30 & 0.35 & 1.14 \\
E-glass & 72.00 & 29.50 & 0.22 & 2.55 \\
E-CR glass & 80.00 & 32.80 & 0.22 & 2.62 \\
\hline
\end{tabular}

Table 2. Description of GFRP plates

\begin{tabular}{lcc}
\hline Plate type & $\begin{array}{c}\text { No. of layers/ type of } \\
\text { reinforcement }\end{array}$ & $\begin{array}{c}\text { Dimensions (mm) } \\
\text { (width } \mathrm{x} \text { height } \mathrm{x} \text { avg. thickness) }\end{array}$ \\
\hline MD.1/MD.2 & 4/MD glass & $480 \times 480 \times 3.27 / 480 \times 480 \times 3.74$ \\
W50.50.1/W50.50.2 & 8/W50.50 glass & $460 \times 460 \times 3.33 / 480 \times 480 \times 3.53$ \\
W60.40.1/W60.40.2 & 6/W60.40 glass & $410 \times 410 \times 3.05 / 410 \times 410 \times 3.06$ \\
W70.30.1/W70.30.2 & 6/W70.30 glass & $400 \times 400 \times 3.36 / 400 \times 400 \times 3.35$ \\
W80.20.1/W80.20.2 & 8/W80.20 glass & $410 \times 410 \times 3.62 / 410 \times 410 \times 3.51$ \\
CFM.1/CFM.2 & 6/CFM glass & $420 \times 420 \times 7.50 / 420 \times 420 \times 6.99$ \\
\hline
\end{tabular}

Table 3. Elastic properties of GFRP plates

\begin{tabular}{lcccccccccc}
\hline Plate & $\begin{array}{c}\mathrm{f} \\
\text { type }\end{array}$ & $\begin{array}{c}\mathrm{E}_{1} \\
(\mathrm{GPa})\end{array}$ & $\begin{array}{c}\mathrm{E}_{2} \\
(\mathrm{GPa})\end{array}$ & $\begin{array}{c}\mathrm{E}_{3} \\
(\mathrm{GPa})\end{array}$ & $\begin{array}{c}\mathrm{G}_{12} \\
(\mathrm{GPa})\end{array}$ & $\begin{array}{c}\mathrm{G}_{13} \\
(\mathrm{GPa})\end{array}$ & $\begin{array}{c}\mathrm{G}_{23} \\
(\mathrm{GPa})\end{array}$ & $\begin{array}{c}v_{12} \\
(-)\end{array}$ & $\begin{array}{c}v_{13} \\
(-)\end{array}$ & $\begin{array}{c}v_{23} \\
(-)\end{array}$ \\
\hline MD.1 & 0.38 & 15.45 & 15.45 & 7.54 & 4.35 & 1.85 & 1.85 & 0.32 & 0.30 & 0.30 \\
W50.50.1 & 0.37 & 17.79 & 17.79 & 7.25 & 2.62 & 1.83 & 1.83 & 0.19 & 0.33 & 0.33 \\
W60.40.1 & 0.39 & 22.30 & 14.86 & 7.57 & 1.76 & 2.00 & 1.73 & 0.23 & 0.32 & 0.33 \\
W70.30.1 & 0.36 & 24.28 & 10.41 & 7.07 & 2.56 & 2.07 & 1.58 & 0.27 & 0.32 & 0.34 \\
W80.20.1 & 0.35 & 27.03 & 6.76 & 6.90 & 2.51 & 2.18 & 1.48 & 0.31 & 0.31 & 0.34 \\
CFM.1 & 0.18 & 9.34 & 9.34 & 4.68 & 3.51 & 1.54 & 1.54 & 0.33 & 0.30 & 0.30 \\
\hline
\end{tabular}


Table 4. Load and displacement values for initiation and maximums

\begin{tabular}{lccccc}
\hline \multirow{2}{*}{ Plate } & \multicolumn{2}{c}{ Initiation } & & \multicolumn{2}{c}{ Maximum } \\
\cline { 2 - 3 } \cline { 6 - 6 } \cline { 5 - 6 } MD.1 & Disp. $(\mathrm{mm})$ & Load $(\mathrm{kN})$ & & Disp. $(\mathrm{mm})$ & Load $(\mathrm{kN})$ \\
\hline MD.2 & 11.13 & 2.31 & & 29.63 & 7.04 \\
W50.50.1 & 7.98 & 1.66 & & 34.27 & 7.93 \\
\hline W50.50.2 & 5.10 & 1.27 & & 33.04 & 6.60 \\
W60.40.1 & 7.61 & 1.03 & & 32.40 & 5.60 \\
\hline W60.40.2 & 6.14 & 1.42 & & 28.74 & 5.15 \\
W70.30.1 & 5.83 & 1.03 & & 18.22 & 3.87 \\
W70.30.2 & 6.54 & 1.11 & & 19.43 & 4.38 \\
\hline W80.20.1 & 4.04 & 0.61 & & 12.41 & 2.62 \\
W80.20.2 & 4.10 & 0.72 & & 14.88 & 3.08 \\
\hline CFM.1 & 3.79 & 1.89 & & 22.97 & 10.05 \\
CFM.2 & 6.29 & 3.24 & & 24.67 & 11.14 \\
\hline
\end{tabular}




\section{Figures:}

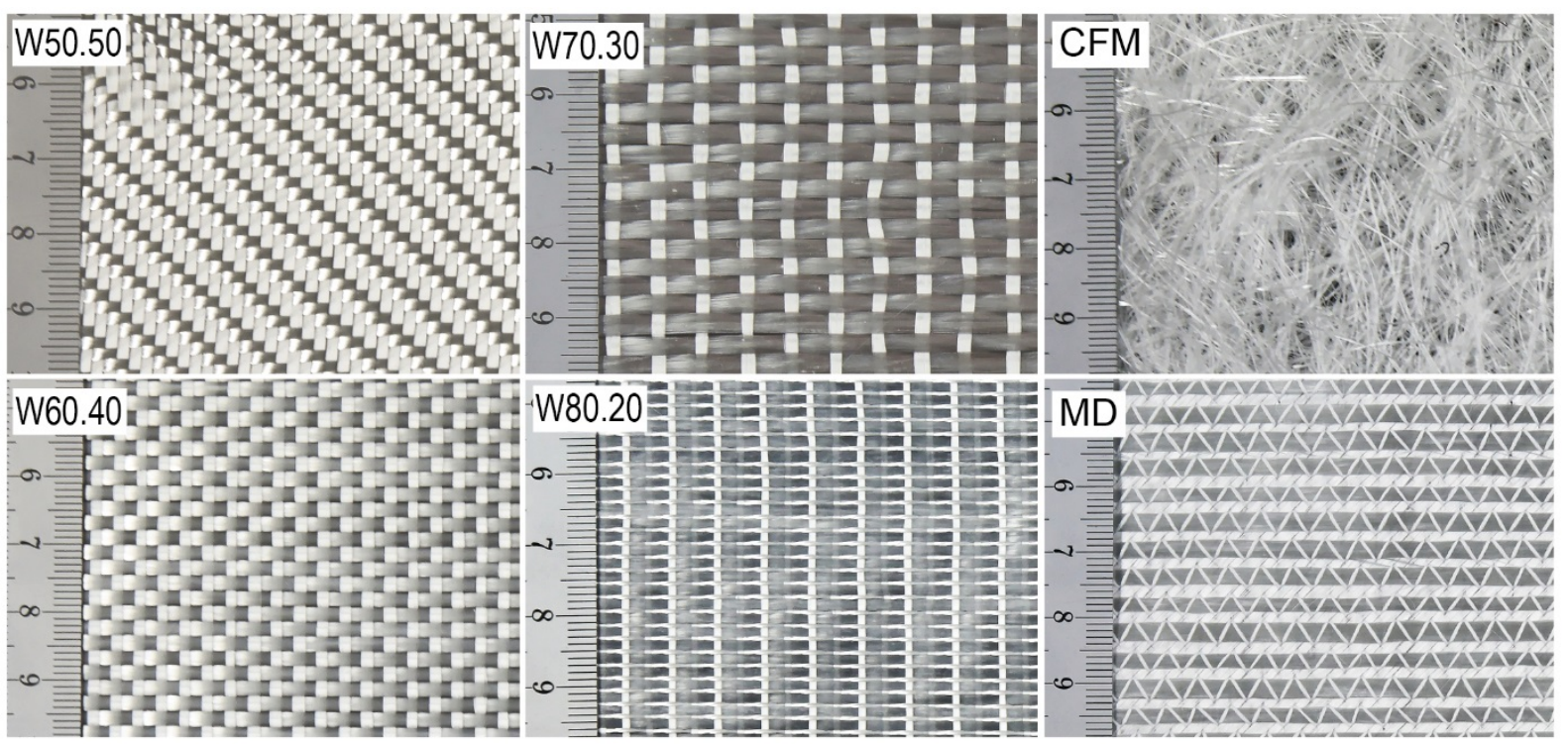

Fig. 1. Detail of glass fiber reinforcements used.

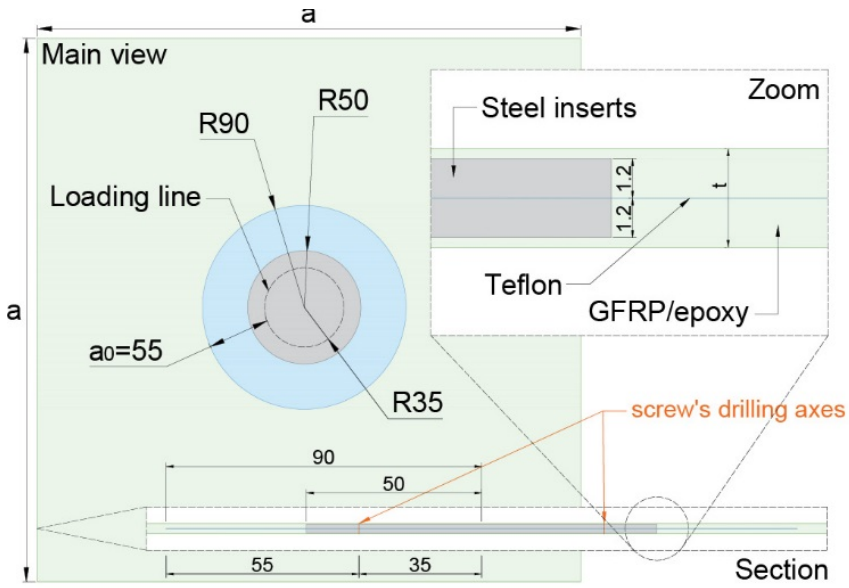

Fig. 2. GFRP plate configuration; dimensions in $\mathrm{mm}$. 


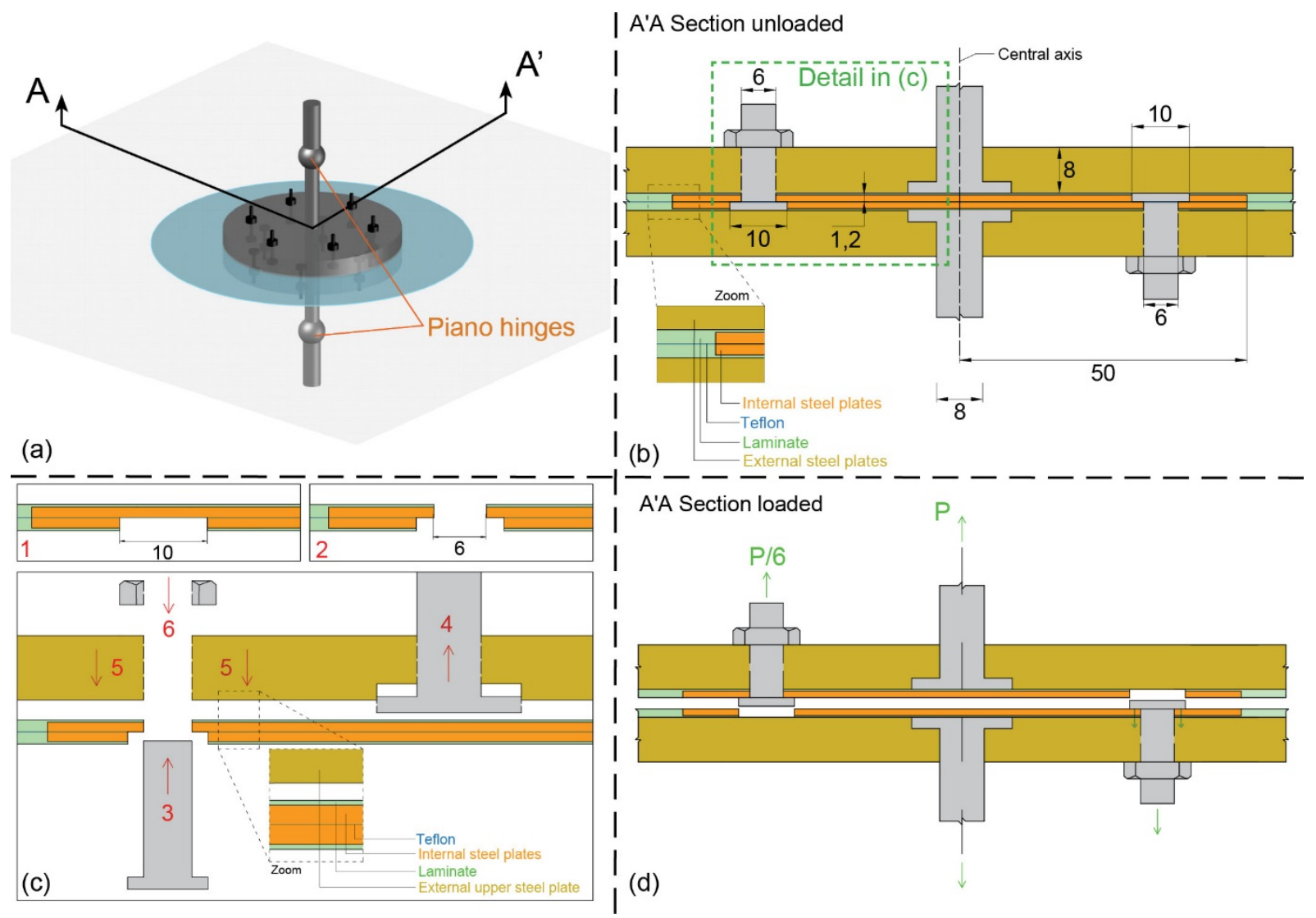

Fig. 3. Assembling of loading system; (a) general 3D view; (b) A'A section (unloaded/closed) view; (c) detailed description of assembling sequence; (d) A'A section (loaded/open).

Dimensions in $\mathrm{mm}$. 

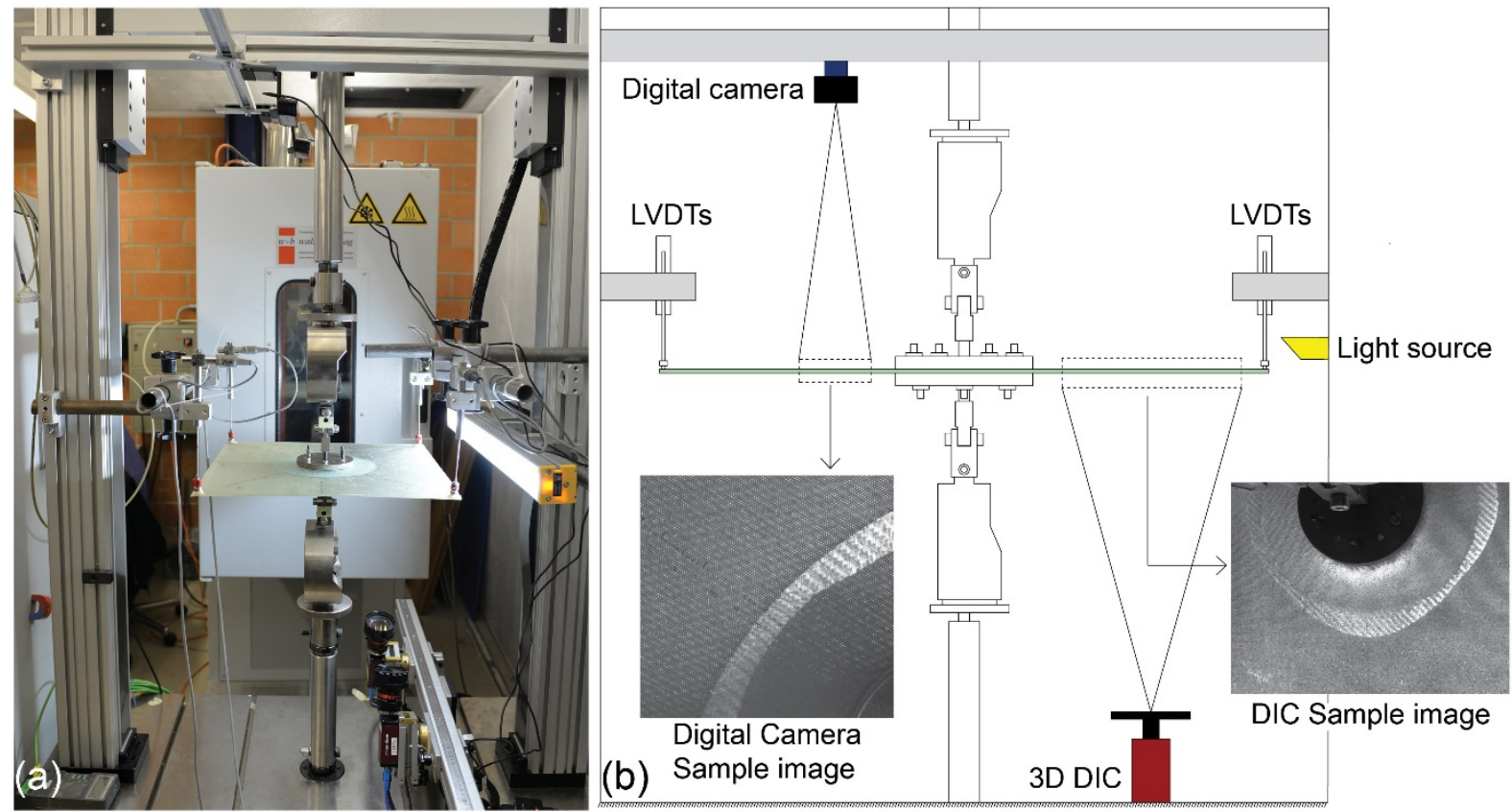

Fig. 4. (a) Experimental set-up and (b) instrumentation layout

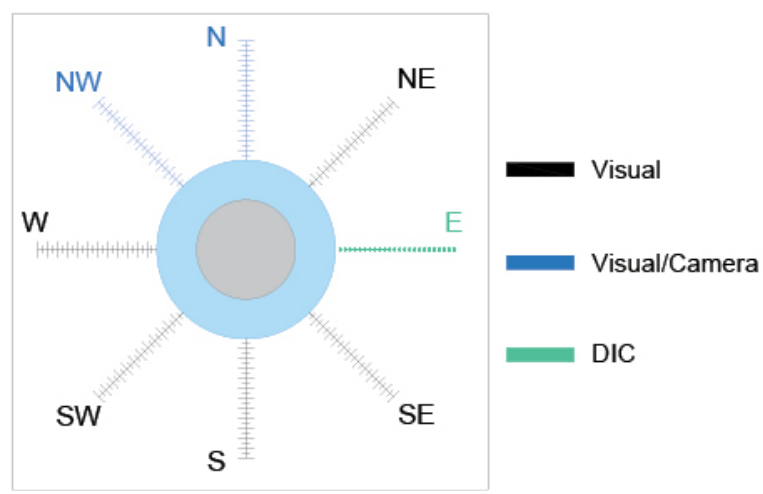

Fig. 5. Layout of crack measuring system. N, S, E and W are the orthogonal directions and NE, SE, NW and SW the diagonal directions. 

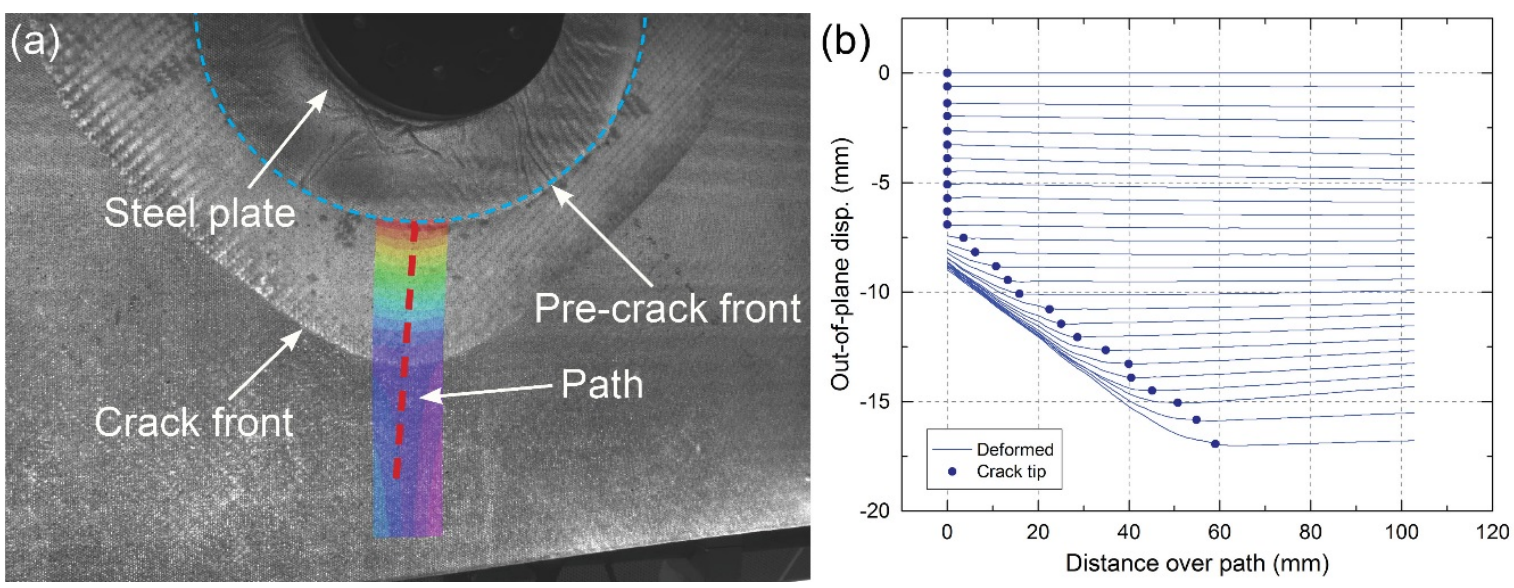

Fig. 6. DIC measurements of plate W50.50.1; (a) example image of area and path of interest; (b) evolution of out-of-plane deformed profiles and location of crack tip.
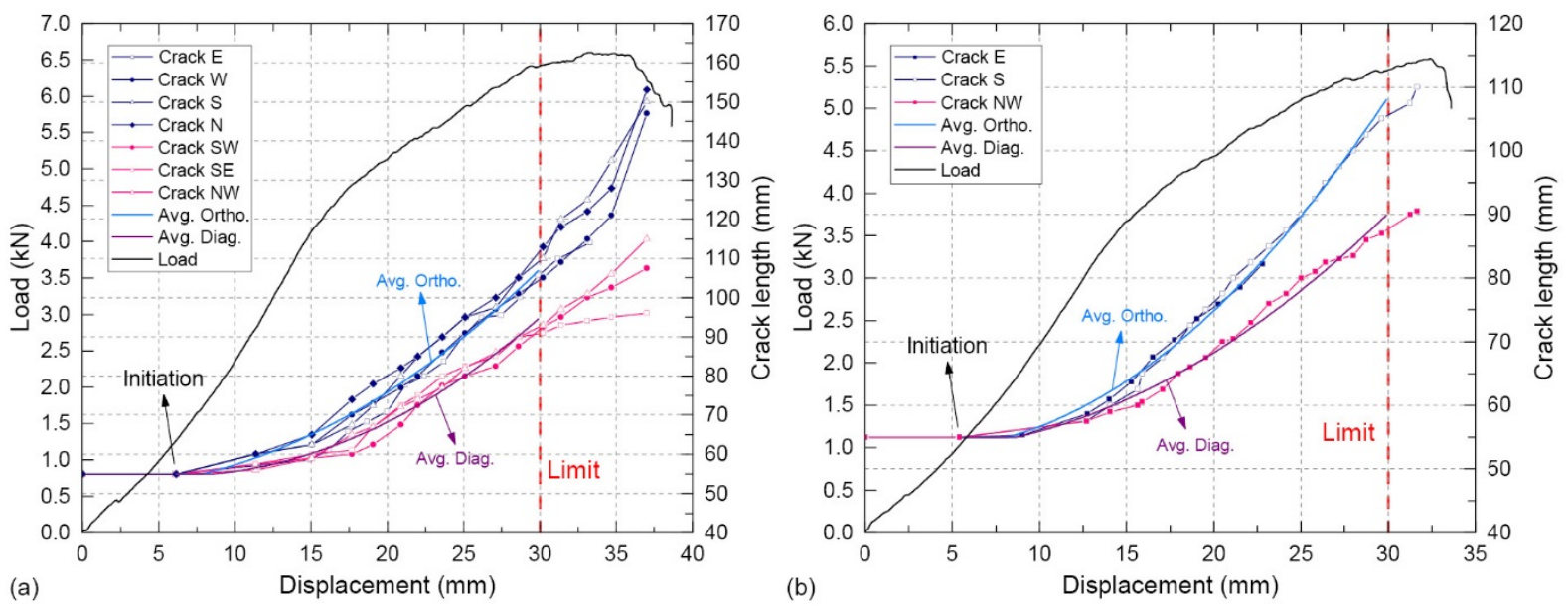

Fig.7. Load and crack lengths vs opening displacement curves; (a) W50.50.1; (b) W50.50.2. 

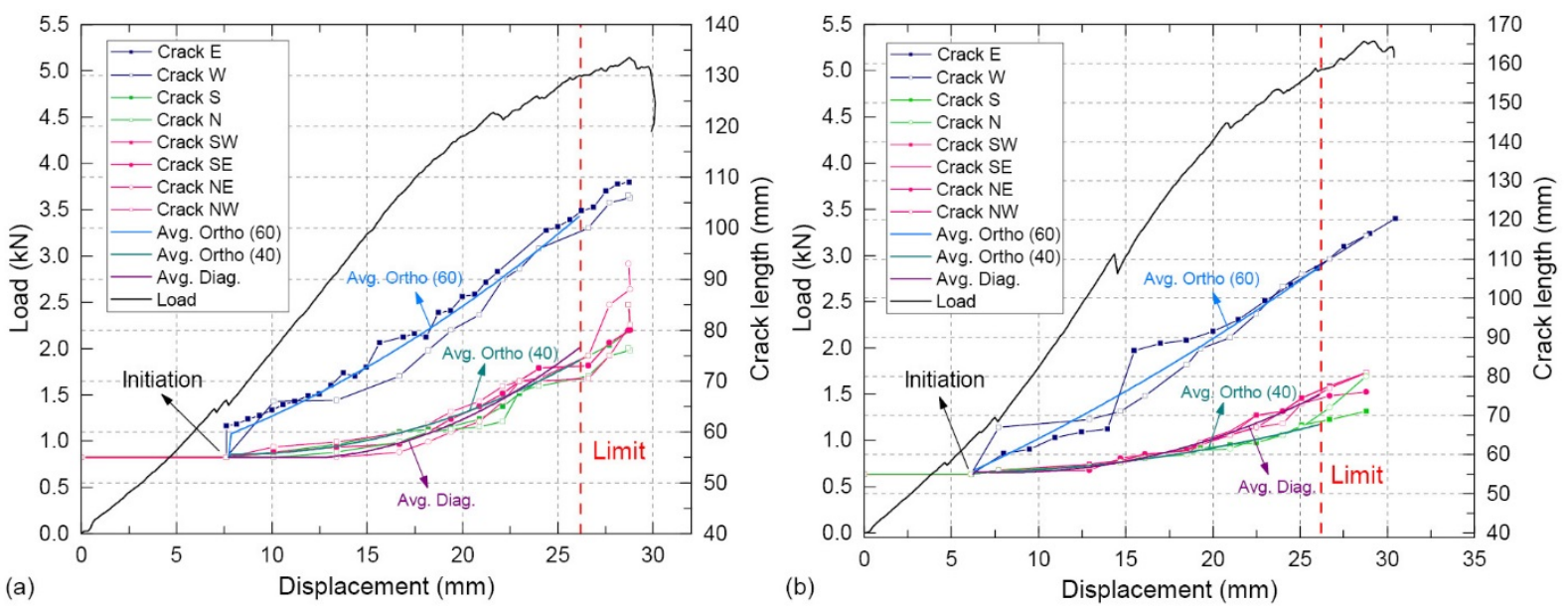

Fig. 8. Load and crack lengths vs opening displacement curves; (a) W60.40.1; (b) W60.40.2.
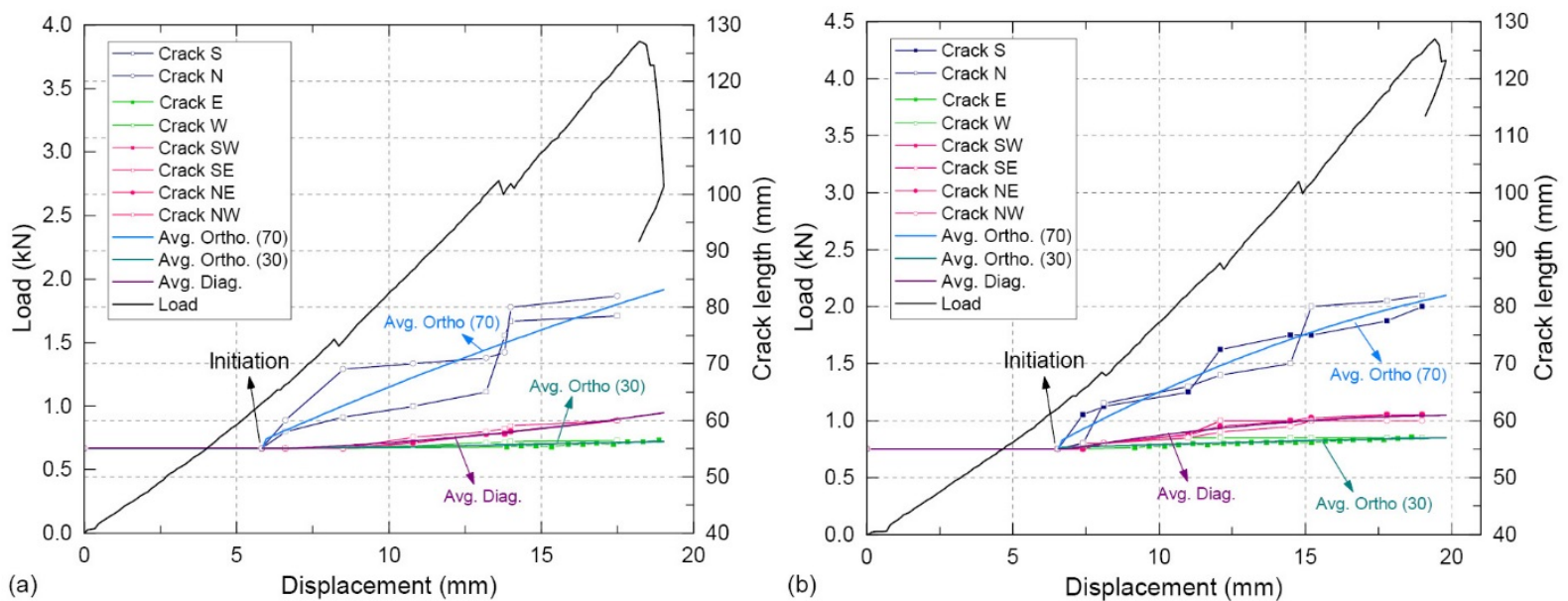

Fig. 9. Load and crack lengths vs opening displacement curves; (a) W70.30.1; (b) W70.30.2.
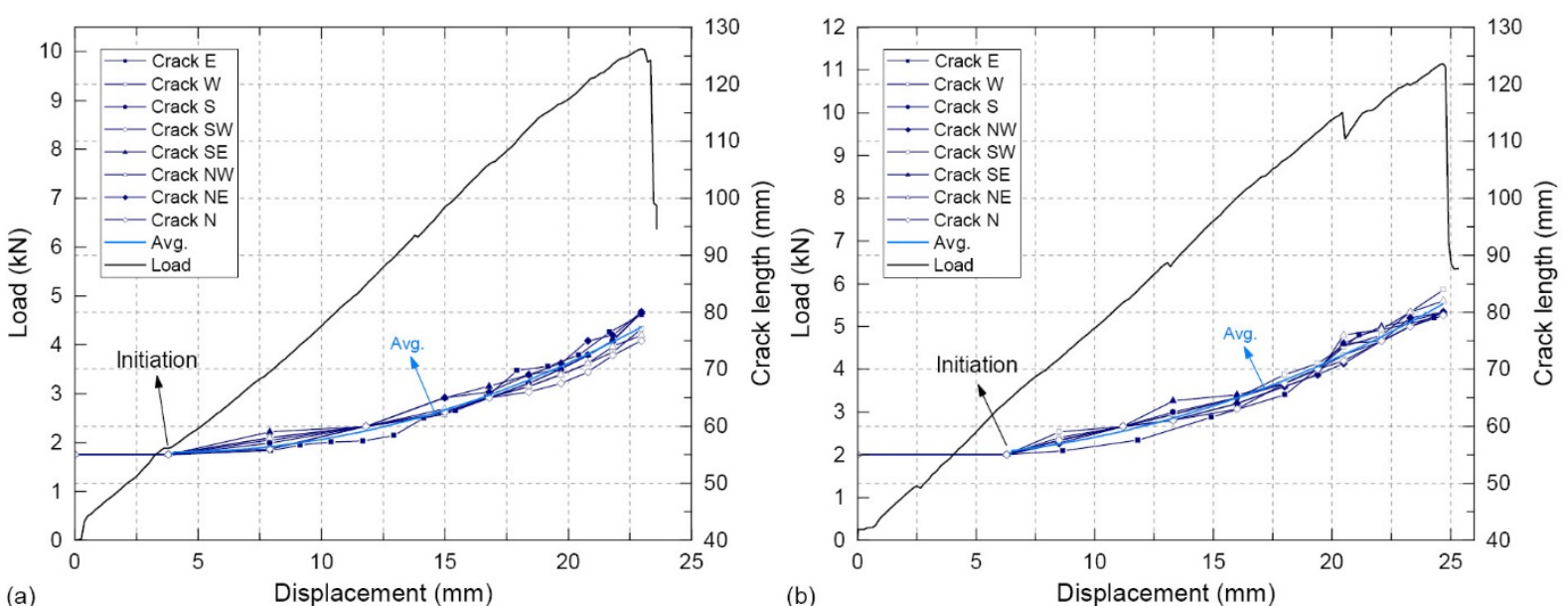

Fig.10. Load and crack lengths vs opening displacement curves; (a) CFM.1; (b) CFM.2. 

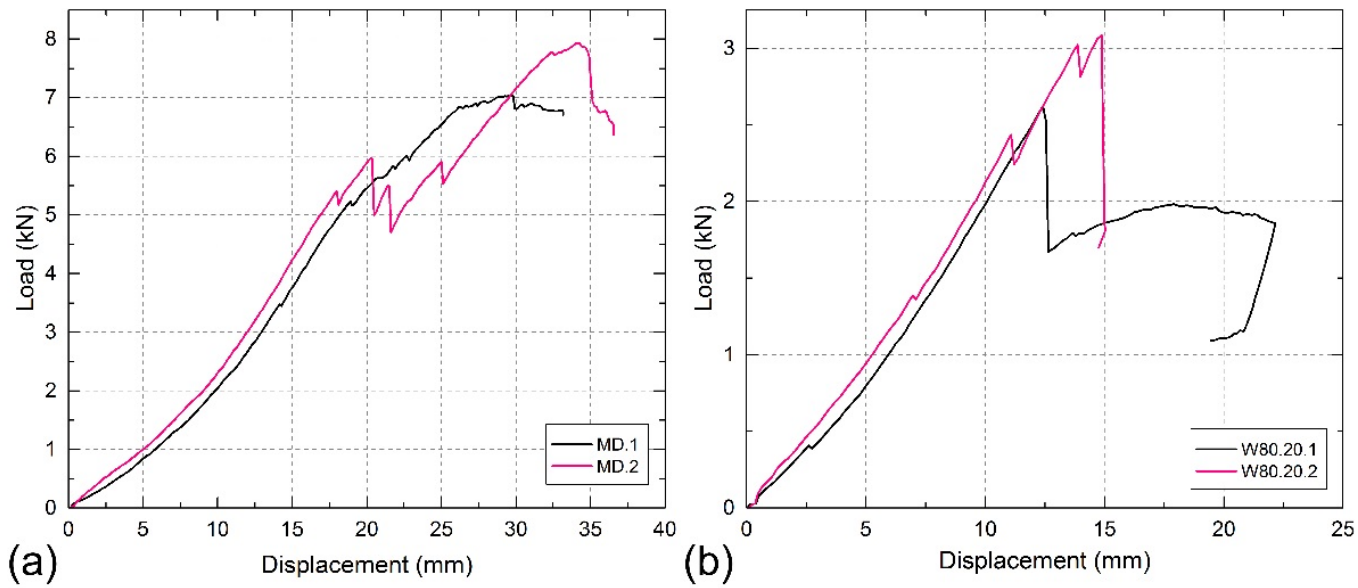

Fig. 11. Load vs opening displacement curves; (a) MD pair; (b) W80.20 pair.

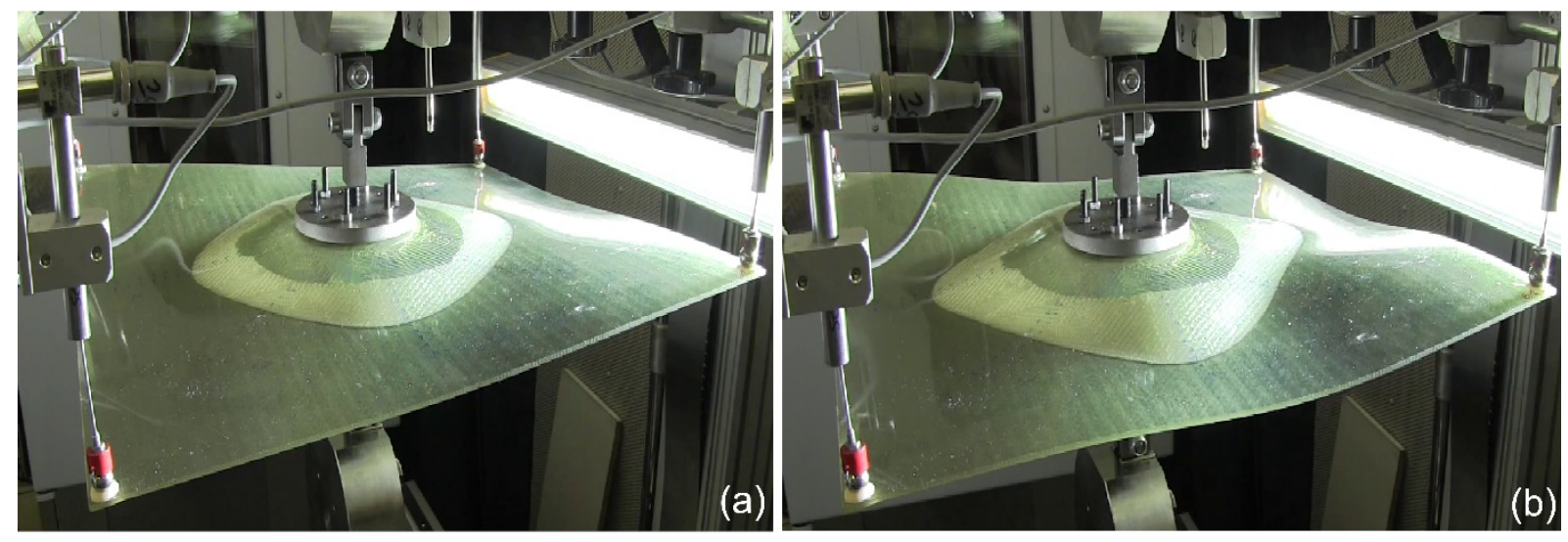

Fig. 12. Example of deformation in plate W50.50.1 at (a) limit of symmetric propagation and (b) beyond limit. 


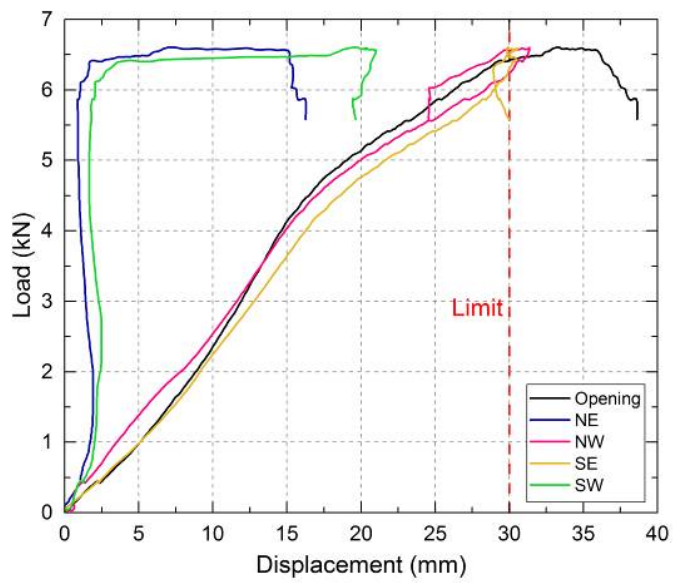

Fig. 13. Displacement of LVDTs at corners of plate W50.50.1 vs load.
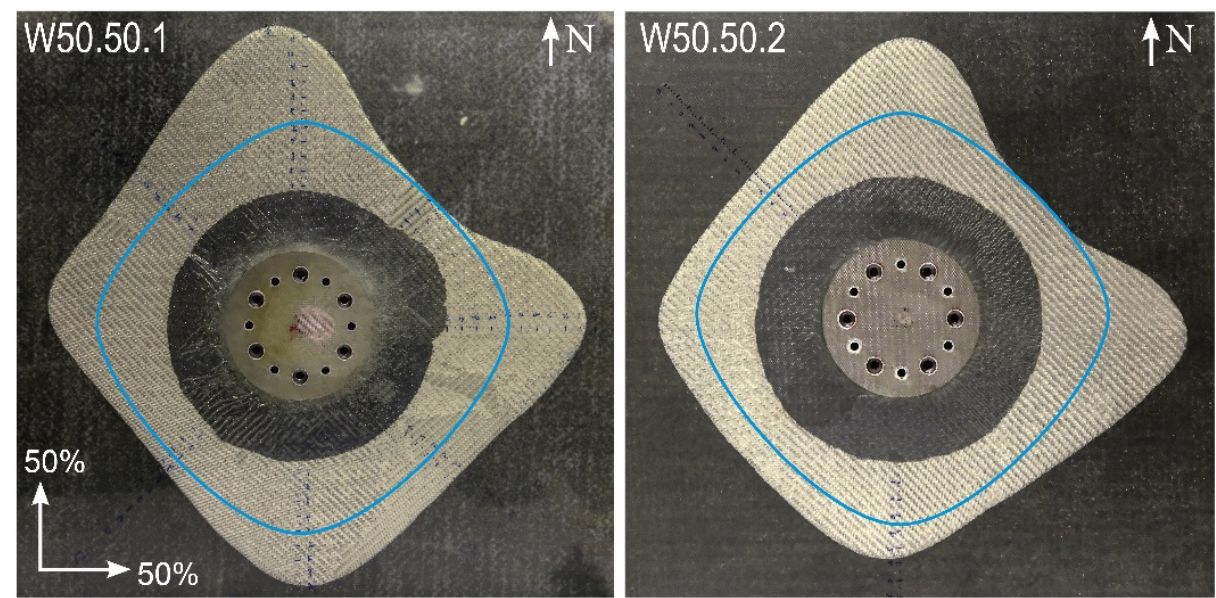

Fig. 14. Crack propagation pattern for W50.50 pair (limit of symmetric propagation marked in blue) 

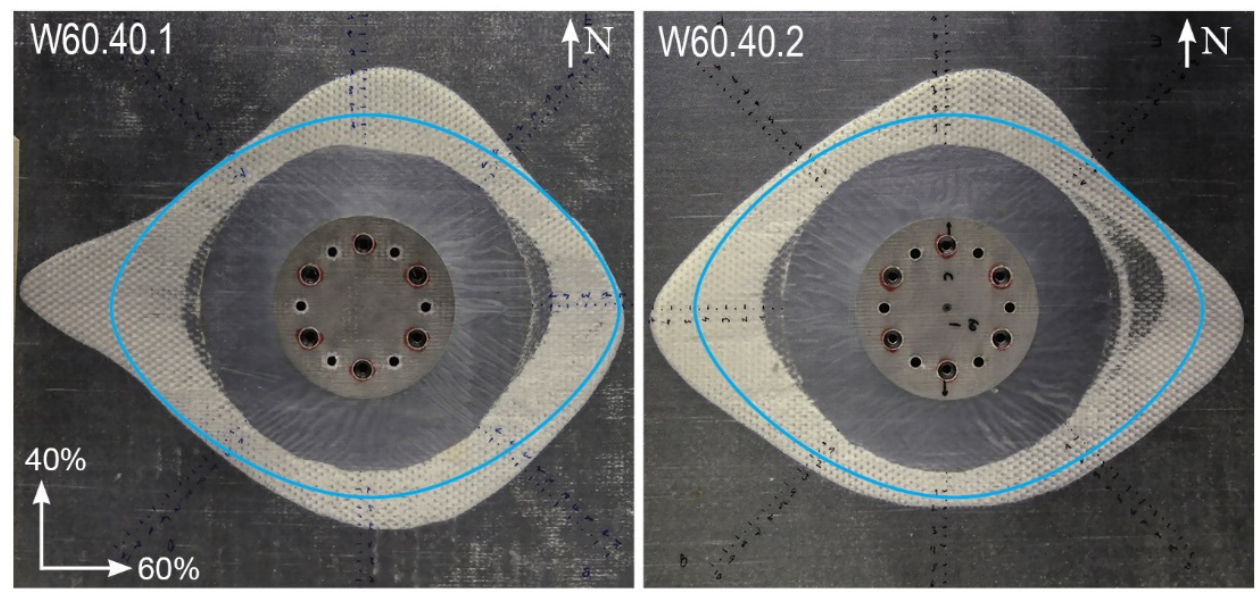

Fig. 15. Crack propagation pattern for W60.40 pair (limit of symmetric propagation marked in blue)

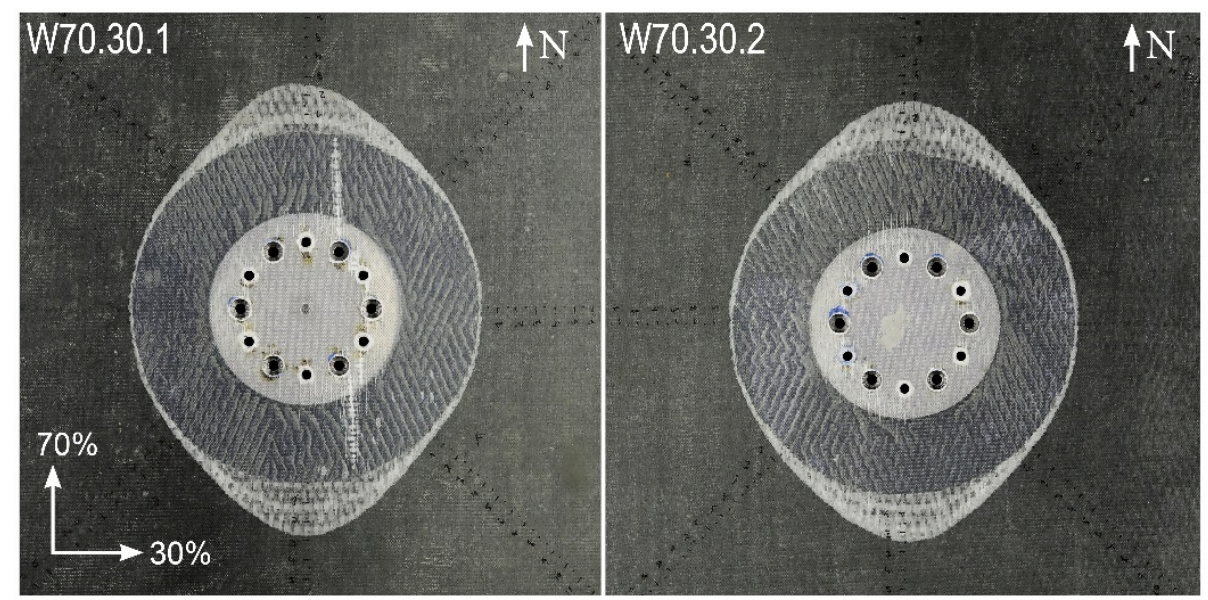

Fig. 16. Crack propagation pattern for W70.30 pair
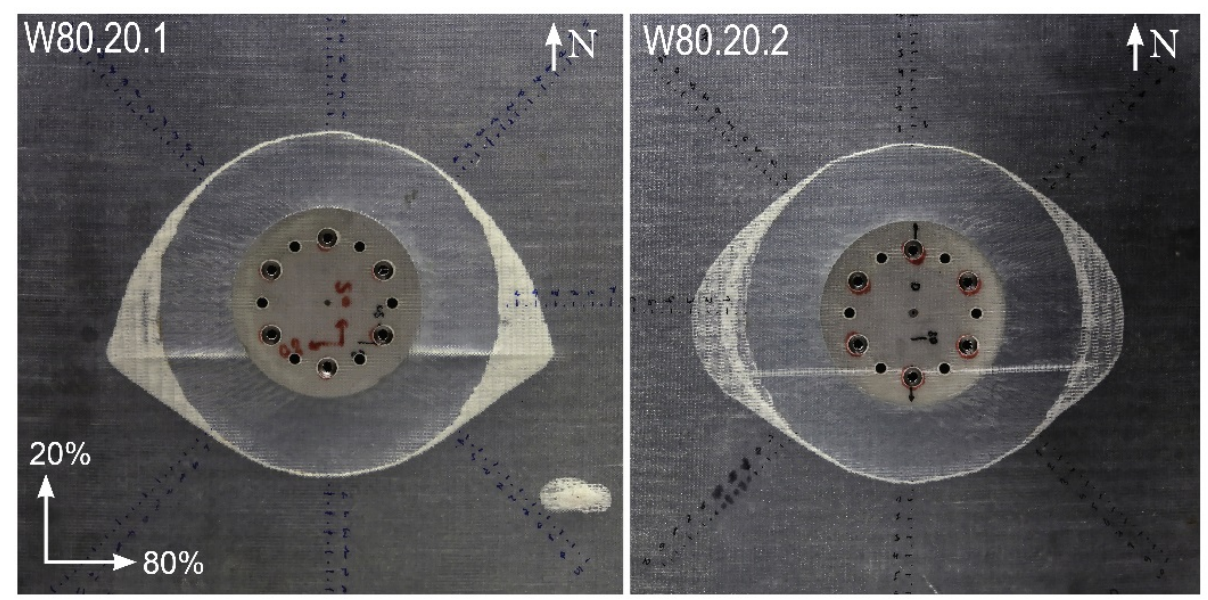

Fig. 17. Crack propagation pattern for W80.20 pair 

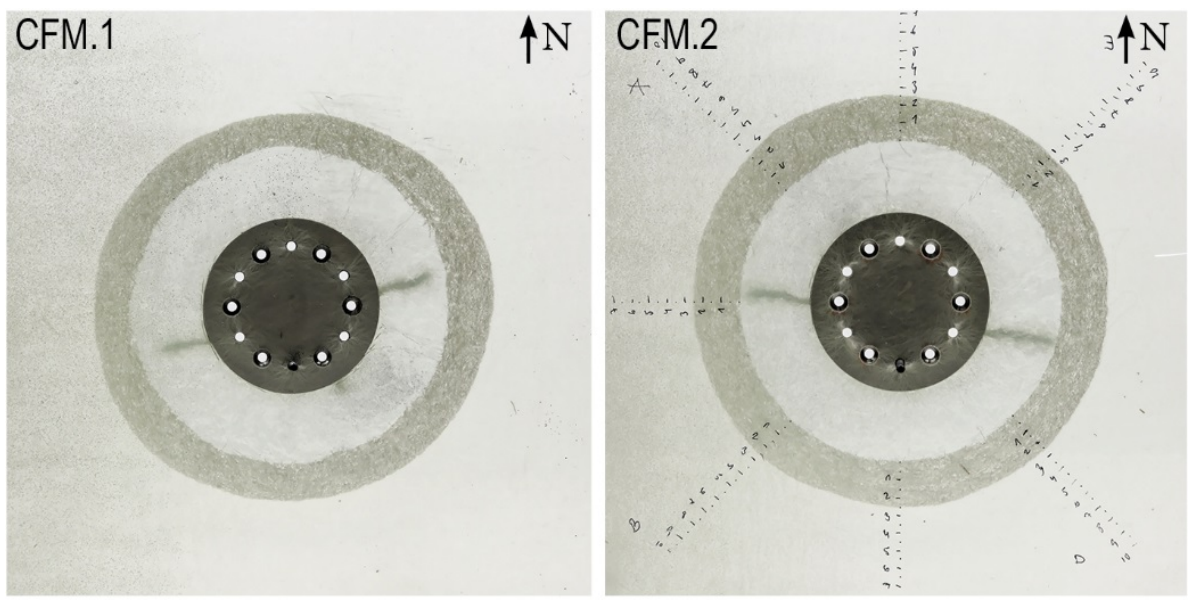

Fig. 18. Crack propagation pattern for CFM pair

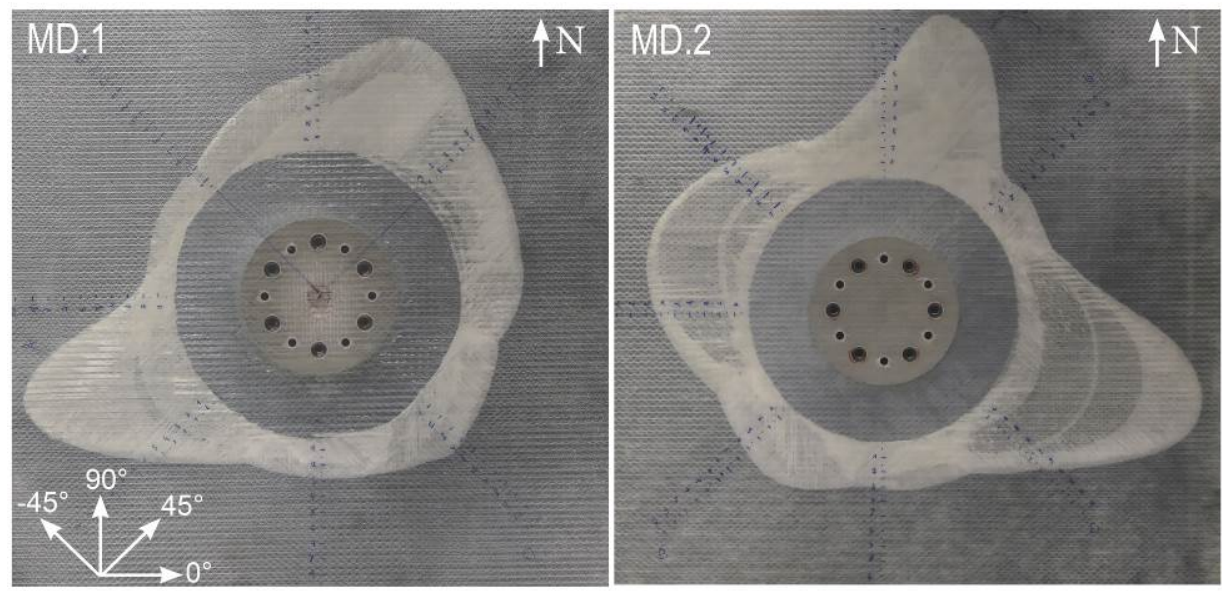

Fig. 19. Crack propagation pattern for MD pair 


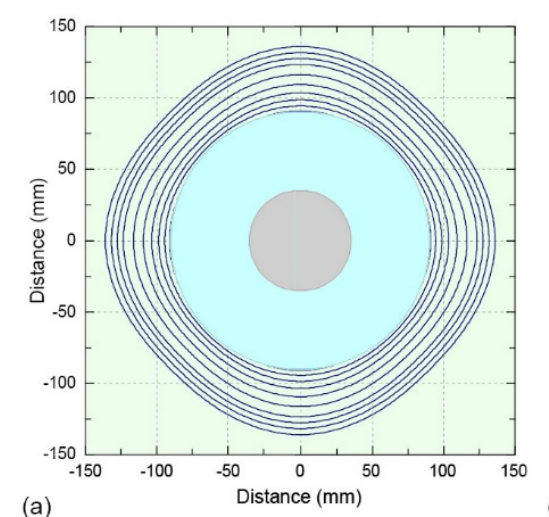

(a)

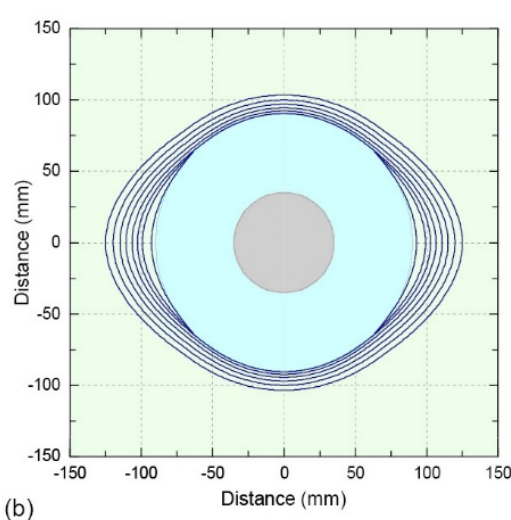

(b)

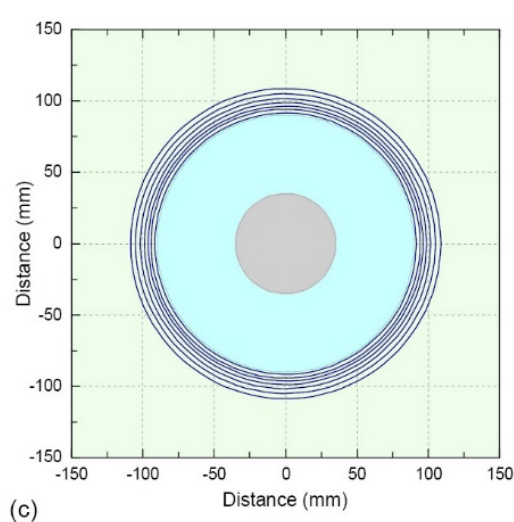

(c)

Fig. 20. Progressive back-calculated propagation perimeters for (a) W50.50.1, (b) W60.40.1 and (c) CFM.1.

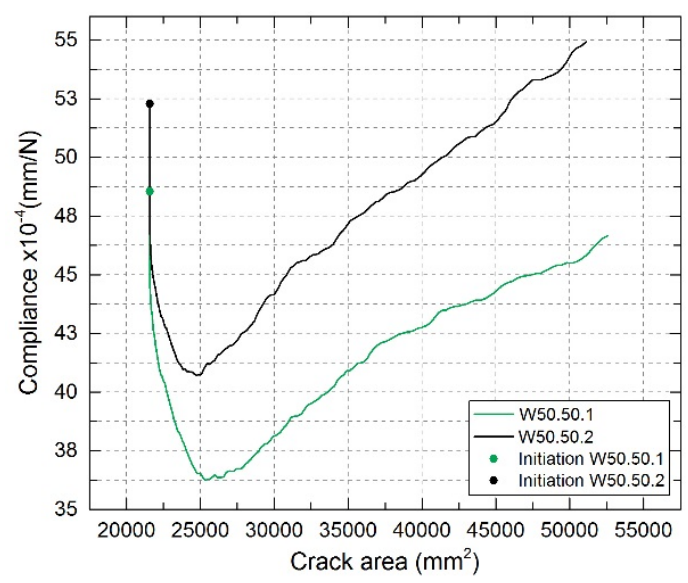

Fig. 21. Compliance vs crack area for W50.50 pair.

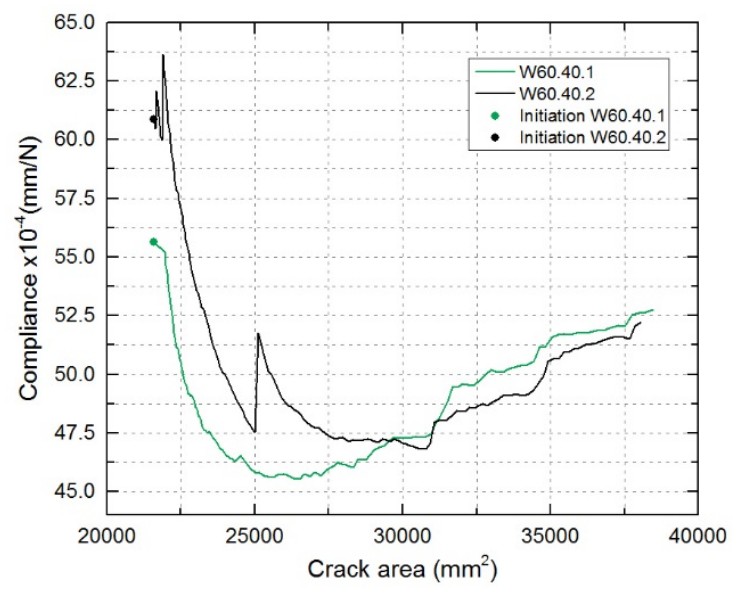

Fig. 22. Compliance vs crack area for W60.40 pair. 


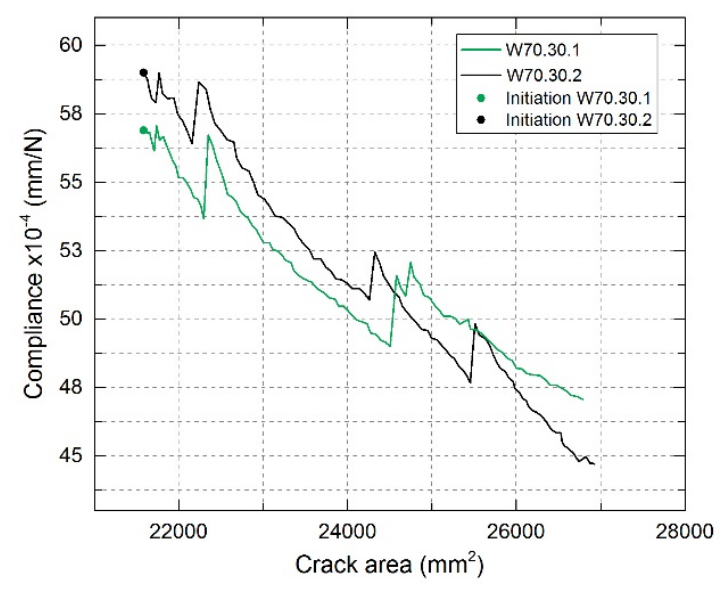

Fig. 23. Compliance vs crack area for W70.30 pair.

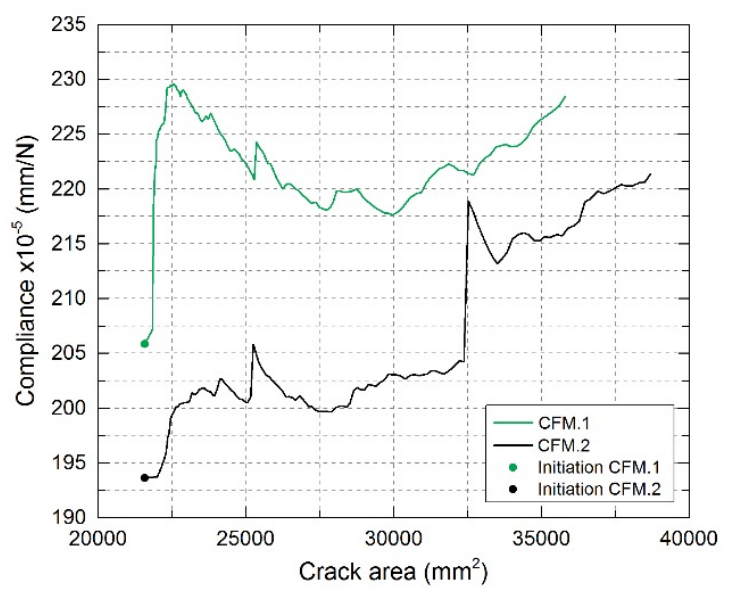

Fig. 24. Compliance vs crack area for CFM pair.
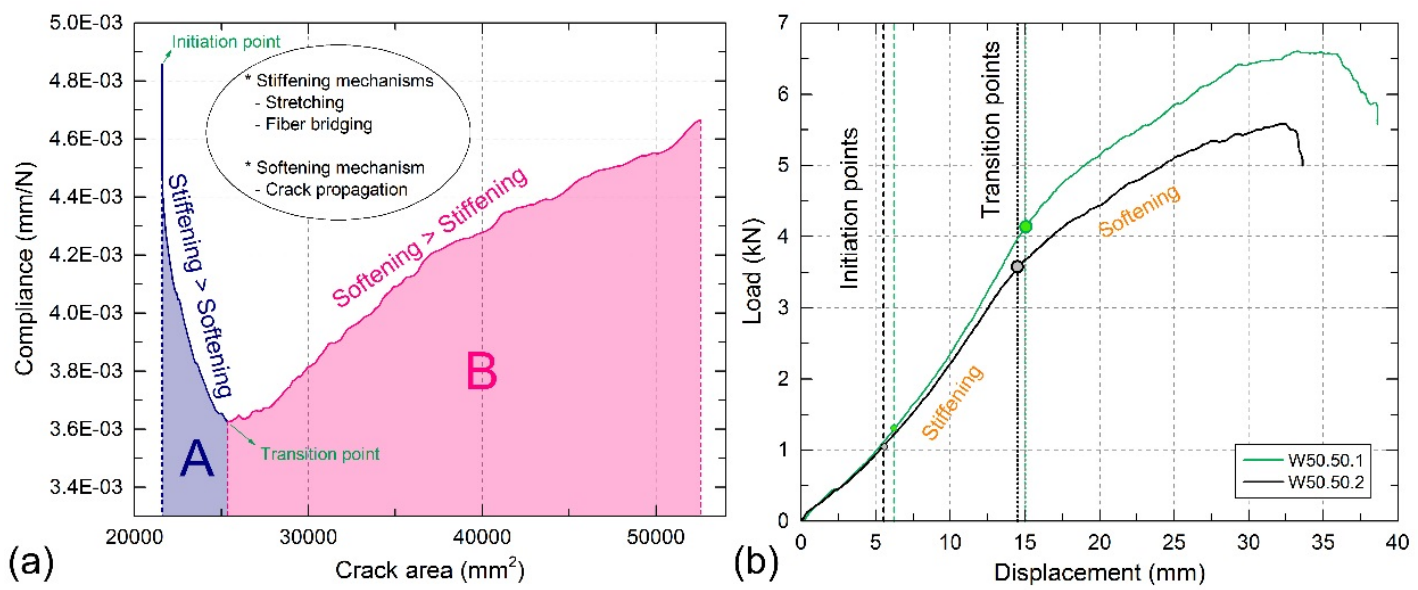

Fig. 25. Identification of stiffening and softening regions on: (a) Compliance vs crack area of W50.50.1; (b) load-displacement curves of plates W50.50.1 and W50.50.2. 


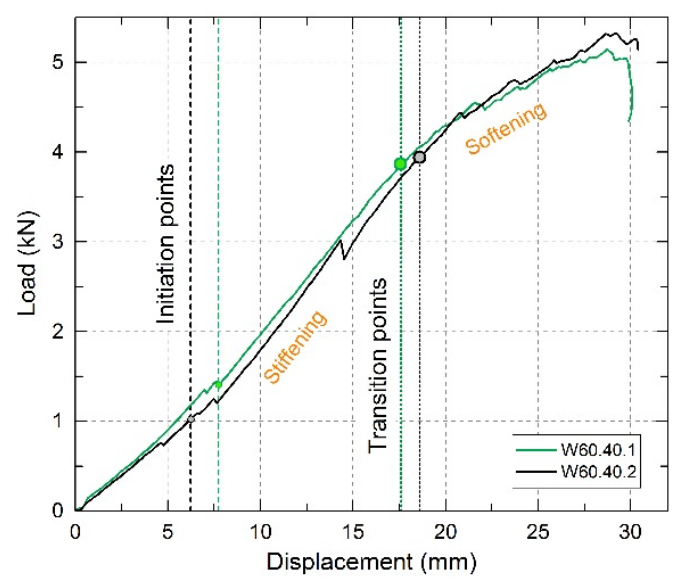

Fig. 26. Identification of stiffening and softening regions on load-displacement curves of W60.40 pair.

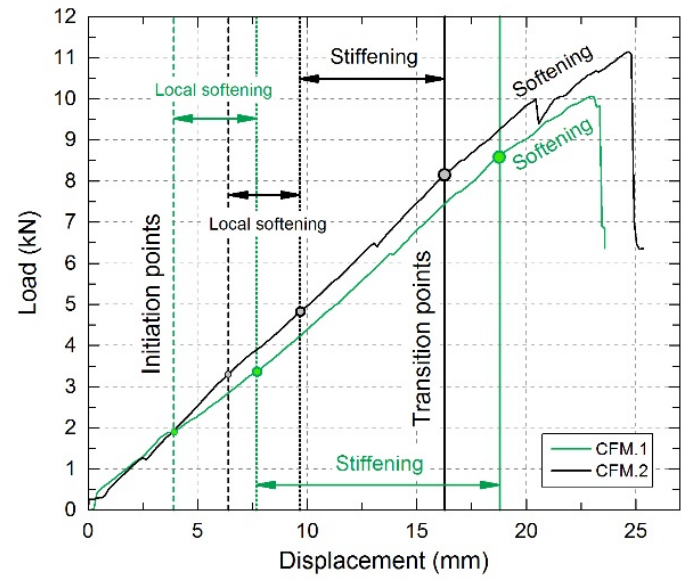

Fig. 27. Identification of stiffening and softening regions on load-displacement curves of CFM pair. 


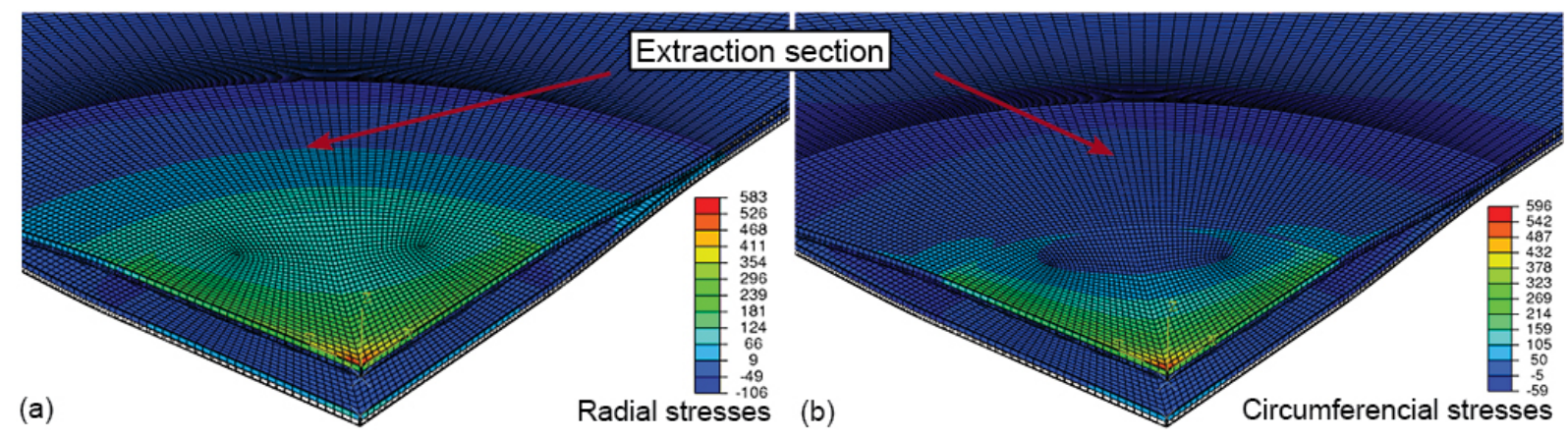

Fig. 28. FE model stress results for plate W50.50.1; (a) total radial stresses; (b) total circumferential stresses $(\mathrm{MPa})$.

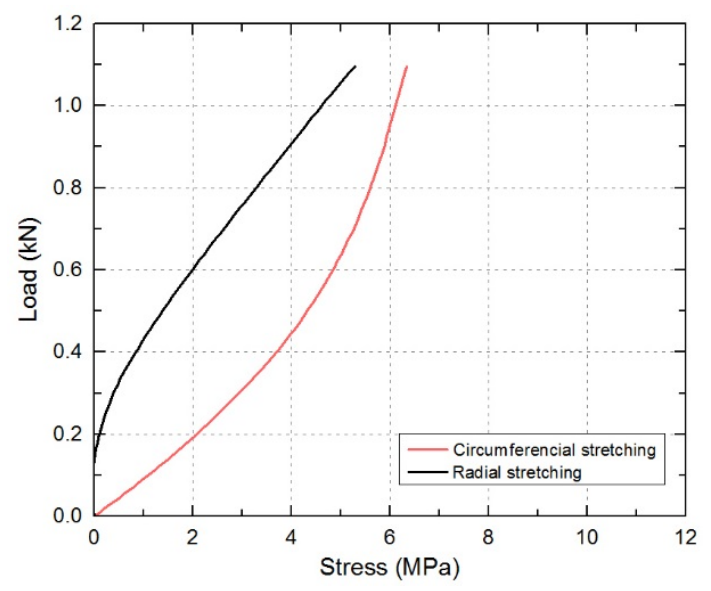

Fig. 29. Load vs stretching stresses obtained at measured section (Fig. 28) from FE model. 